Article

\title{
Comparative Analysis of Far East Sikhotinsky Rhododendron (Rh. sichotense) and East Siberian Rhododendron (Rh. adamsii) Using Supercritical $\mathrm{CO}_{2}$-Extraction and HPLC-ESI-MS/MS Spectrometry
}

\author{
Mayya Razgonova ${ }^{1,2, *(D)}$, Alexander Zakharenko ${ }^{1,2}(\mathbb{D})$, Sezai Ercisli ${ }^{3}$ (D), Vasily Grudev ${ }^{4}$ and \\ Kirill Golokhvast $1,2,5$ \\ 1 N.I. Vavilov All-Russian Institute of Plant Genetic Resources, 190000 Saint-Petersburg, Russia; \\ rarf@yandex.ru (A.Z.); k.golokhvast@vir.nw.ru (K.G.) \\ 2 SEC Nanotechnology, Far Eastern Federal University, 690950 Vladivostok, Russia \\ 3 Agricultural Faculty, Department of Horticulture, Ataturk University, 25240 Erzurum, Turkey; \\ sercisli@gmail.com \\ 4 Far Eastern Investment and Export Agency, 123112 Moscow, Russia; grudev@ya.ru \\ 5 Pacific Geographical Institute, Far Eastern Branch of the Russian Academy of Sciences, \\ 690041 Vladivostok, Russia \\ * Correspondence: m.razgonova@vir.nw.ru
}

Academic Editors: Seung Hwan Yang and Satyajit Sarker

Received: 29 June 2020; Accepted: 12 August 2020; Published: 19 August 2020

\begin{abstract}
Rhododendron sichotense Pojark. and Rhododendron adamsii Rheder have been actively used in ethnomedicine in Mongolia, China and Buryatia (Russia) for centuries, as an antioxidant, immunomodulating, anti-inflammatory, vitality-restoring agent. These plants contain various phenolic compounds and fatty acids with valuable biological activity. Among green and selective extraction methods, supercritical carbon dioxide $\left(\mathrm{SC}-\mathrm{CO}_{2}\right)$ extraction has been shown to be the method of choice for the recovery of these naturally occurring compounds. Operative parameters and working conditions have been optimized by experimenting with different pressures (300-400 bar), temperatures $\left(50-60^{\circ} \mathrm{C}\right)$ and $\mathrm{CO}_{2}$ flow rates $(50 \mathrm{~mL} / \mathrm{min})$ with $1 \%$ ethanol as co-solvent. The extraction time varied from 60 to $70 \mathrm{~min}$. A HPLC-UV-VIS-ESI-MS/MS technique was applied to detect target analytes. A total of 48 different biologically active components have been identified in the Rh. adamsii SC- $\mathrm{CO}_{2}$ extracts. A total of 31 different biologically active components have been identified in the $R h$. sichotense $\mathrm{SC}-\mathrm{CO}_{2}$ extracts.
\end{abstract}

Keywords: Rhododendron sichotense; Rhododendron adamsii; supercritical fluid extraction; HPLC-MS/MS; phenolic compounds

\section{Introduction}

A total of 19 species of rhododendrons are growing in the territory of Russia, the main part of which (13 species) are found only in the flora of the Russian Far East and Eastern Siberia [1]. Russian researchers classify the genus Rhododendron somewhat differently than foreign ones [2]. At present, there is no unified classification scheme for a taxon, since the genus is very large-more than 800 species, as well as the presence of a large number of convergent characters among its representatives, complicating the construction of a natural classification [3].

The genus system, developed and adopted by Russian scientists, divides the genus Rhododendron into subgenera and series. In it, Rhododendron adamsii Rehder and Rhododendron parvifolium Adams are assigned to the subgenus Osmothamnus Maximowicz (Fragrantia E. Busch series and Parvifolia E. 
Busch series) (Table 1). Species of Rh. dauricum L., Rh. ledebourii Pojarkova, Rh. sichotense Pojarkova and Rh. micronulatum Turczaninowia constitute the series Daurica Pojarkova subgenus Rhodorastrum (Maxim.) Drude [4].

Rhododendron sichotense Pojark. is a plant from the genus of rhododendrons [5]. It grows in the Far East (Primorsky Krai) on the eastern slope of the Sikhote Alin ridge. This type of rhododendron is included in the Red Book of the Russian Federation [6]. From its closest relatives, Rhododendron dauricum and Rhododendron micronulatum is distinguished by larger, sometimes more than $7 \mathrm{~cm}$ wide, flowers and wider, green leaves from the underside, not falling leaves. Large flowers, lush foliage, winter hardiness suggest that $R h$. sichotense is a very promising garden plant for areas with a harsh climate [7].

Table 1. Classification of genus Rhododendron L.

\begin{tabular}{cccc}
\hline No. & Species or Variety & Subgenus & Row \\
\hline 1 & $\begin{array}{c}\text { Rh. sichotense Pojark.; Rh. micronulatum Pojark.; Rh. } \\
\text { dauricum L.; Rh. ledebourii Pojark. }\end{array}$ & Rhodorastrum & Daurica Pojark. \\
\hline 2 & Rh. parvifolium Adams [Rh. lapponicum (L.) \\
Wahlenb.] & $\begin{array}{c}\text { Osmothamnus } \\
\text { Maximowicz }\end{array}$ & Parvifolia E. Busch \\
\hline 3 & Rh. adamsii Rehd. [Rh. fragrans (Adams) Maxim.] & & Fragrantia E. Busch \\
\hline
\end{tabular}

Rh. adamsii Rheder is a shrub found in Eastern Siberia and Baikal in the alpine and subalpine zones of the mountains, forming a shrub tundra, and at the upper border of the forest at an altitude of 1200-2500 $\mathrm{m}$ above sea level. This is a shrub from 1 to $3 \mathrm{~m}$ in height with falling leaves. The flowers are pink, large enough, and open before the leaves appear. The leaves are narrow, green above, grayish green below, and very fragrant. $R h$. adamsii grows in pine and deciduous forests with grass and moss, on forest edges and especially on rocky mountains [8].

Traditional medicine uses different types of rhododendron to treat a number of diseases of the respiratory system, the gastrointestinal tract, chronic skin diseases, hypertension, rheumatism, helminthiases, etc. [9]. The stupefying smell formed during the burning of leaves and caused by the sharp evaporation of volatile terpenes has long been used by indigenous ethnic groups of Siberia and Far East as a psychoactive, analgesic, and narcotic drug [10,11].

Rh. adamsii Rehder is used as a stimulant and tonic by the populations of Buryatia, Mongolia and China. Decoctions and tinctures of it are used for cold diseases, as a diuretic agent for cardiac edema, as well as an adaptogen [12]. Pharmacological studies have shown that $R h$. adamsii has antimicrobial, anti-inflammatory, immunomodulating, antioxidant effects $[13,14]$.

In essential oil of $R h$. adamsii, it is possible to isolate the components present both in the leaves and stems of the plant: $\alpha$ - and $\beta$-pinenes, $\beta$-myrcene, cis- $\beta$-ocimene, isoledene, aromadendrene, humulene, $\beta$-farnesol, $\gamma$-murolene, $\beta$-selinene, ledene, $\alpha$-farnesol, $\delta$-cadinene, trans-nerolidol, spathulenol, $\beta$-elemenone, germacrone [15]. The essential oil of the stems of the plant contains germacrene D and germacrene $\mathrm{B}$, which are absent from the essential oil of the leaves. In almost all samples of essential oil of leaves and stems of $R h$. adamsii there is found 4-phenyl-2-butanone, the content of which is from 3 to $13 \%$, as well as its related 4-phenyl-2-butanol, the content of which is from 1.9 to $7.4 \%$ [16].

This study considers the possibility and effectiveness of supercritical carbon dioxide $\left(\mathrm{SC}-\mathrm{CO}_{2}\right)$ extraction of biologically active substances from stems and leaves of $\mathrm{Rh}$. adamsii. Previously, the authors of this article successfully used $\mathrm{SC}-\mathrm{CO}_{2}$ extraction to obtain biologically active substances from plants of the Far Eastern taiga Panax ginseng, Rhodiola rosea, and Schisandra chinensis, which are extremely popular in traditional medicines of Southeast Asia [17,18].

Supercritical fluid extraction (SFE) has been used since the late 1970s to analyze food products, isolate biologically active substances and determine lipid levels in food, as well as levels of toxic substances [19-21]. The use of SFE for fractionation and/or enrichment of certain components in products has been reported since the 1980s [22-24]. In addition, the products do not have residues of organic solvents, which occur with conventional extraction methods, and solvents can be toxic, 
for example, in the case of methanol and $n$-hexane. Easy solvent removal from the final product, high selectivity and the use of moderate temperatures in the extraction process are the main attractive factors of supercritical technology, leading to a significant increase in research for use in the food and pharmaceutical industries [25-27].

An alternative to the use of co-solvents in the case of poorly soluble or practically insoluble compounds is to completely change the process scheme using the so-called supercritical solvent extraction (SAE). Industrial-scale devices with technological schemes containing $\mathrm{CO}_{2}$ processing plants have already been developed; therefore, most of the solvent/anti-solvent is recovered. SAE increases are associated with the same process conditions as the pressure, temperature and concentration of solutes in the slurry. However, the main parameter is the molar fraction of $\mathrm{CO}_{2}$. It depends on the relative flow rate of the $\mathrm{CO}_{2}$ and the solvent liquid to set the supercritical precipitant composition for the $\mathrm{CO}_{2}$ /solvent mixture used [28].

Popova et al. [29] (2018) investigated the possibility of SC- $\mathrm{CO}_{2}$ extracting chlorophylls and carotenoids of Ledum palustre L. (Rhododendron tomentosum Harmaja) by supercritical fluid extraction using supercritical carbon dioxide and a co-solvent of ethyl alcohol as a solvent. It has been found that by varying the pressure and temperature of the fluid, the duration of processing and the moisture content of the raw material, extracts can be obtained enriched in one or both of the recoverable pigments. Furthermore, in this case, the amount of ethanol used as a co-solvent was 5\% and was necessary and sufficient for efficient extraction of pigments with supercritical $\mathrm{CO}_{2}$.

The anti-inflammatory activity of two extracts from the aerial parts of Ledum palustre L. (Rh. tomentosum Harmaja) has been reported by [30]. The volatile oil was obtained by $\mathrm{SC}-\mathrm{CO}_{2}$ extraction and the essential oil by hydrodistillation (HD). The anti-inflammatory activity was evaluated by the subcutaneous carrageenan injection-induced hind paw oedema. The results show that L. palustre essential oil enhanced a significant inhibition of oedema (50-73\%) for HD oil and (52-80\%) for SFE oil.

The results of SC- $\mathrm{CO}_{2}$-extraction of leaves and branches of rhododendrons, in particular, indicated that, when using this technology, the extract contained all biologically active components of the plant, as well as inert mixtures of extracted compositions. This study is devoted to comparative mass spectrometry of extracted biologically active substances from two closely related subgenus of rhododendron: Rh. sichotense Pojark. and Rh. adamsii Rehder.

\section{Results and Discussion}

In the first instance, the influence of the supercritical parameters $\left(\mathrm{CO}_{2}\right.$ flow rate, temperature, $\%$ co-solvent, and pressure) on extraction yield was investigated. The cumulative quantitative extracts yield are summarized in the Table 2. Several experimental conditions were investigated working in a pressure range of 300-400 bar, with co-solvent $\mathrm{EtOH}$ and a temperature range of $50-60^{\circ} \mathrm{C}$.

Orthogonal projection representing the extraction yield at 300 to 400 Bar and $50-70{ }^{\circ} \mathrm{C}$ is shown in Figure 1. The best results were obtained at 370 Bar and $60^{\circ} \mathrm{C}$. An ion trap amaZon SL BRUKER DALTONIKS equipped with an electron spray ionization (ESI) source in the negative and positive ion modes and analysis of fragmented ions was used in this scientific work.

A screening of biologically active substances from $R h$. adamsii sample and $R h$. sichotense sample was obtained using this method. Typical base peak chromatograms (BPC) of analyzed target analytes are shown in the Supplementary Material. Identification of compounds was assigned by comparison of their UV-Vis spectra and mass spectrometric data obtained under both negative and positive electron spray ionization $\left(\mathrm{ESI}^{-} / \mathrm{ESI}^{+}\right)$conditions and with the scientific literature. Under these conditions a total of 800 peaks were detected in the ion chromatogram. 
Table 2. Extraction yield of $R h$. adamsii presented depending on operational parameters (pressure, temperature, $\mathrm{CO} 2$ flow rate, $\%$ co-solvent).

\begin{tabular}{cccccc}
\hline No. & $\begin{array}{c}\text { Temperature } \\
\left({ }^{\circ} \mathbf{C}\right)\end{array}$ & Pressure (Bar) & $\begin{array}{c}\mathbf{C O}_{2} \text { Flow Rate } \\
(\mathbf{m L} / \mathbf{m i n})\end{array}$ & $\begin{array}{c}\text { \% Co-Solvent } \\
\text { EtOH }\end{array}$ & $\begin{array}{c}\text { Extraction } \\
\text { Yield (mg/g) }\end{array}$ \\
\hline 1 & 50 & 300 & 30 & 1 & 1.23 \\
\hline 2 & 50 & 350 & 50 & 2 & 3.27 \\
\hline 3 & 50 & 370 & 30 & 1 & 3.25 \\
\hline 4 & 50 & 400 & 50 & 2 & 4.15 \\
\hline 5 & 60 & 300 & 30 & 2 & 5.81 \\
\hline 6 & 60 & 350 & 30 & 1 & 7.12 \\
\hline 7 & 60 & 370 & 50 & 1 & 10.86 \\
\hline 8 & 60 & 400 & 30 & 1 & 8.13 \\
\hline 9 & 70 & 300 & 30 & 1 & 7.15 \\
\hline 10 & 70 & 350 & 50 & 1 & 7.28 \\
\hline 11 & 70 & 370 & 30 & 3.10 \\
\hline 12 & 70 & 400 & 30 & 7.90 \\
\hline
\end{tabular}

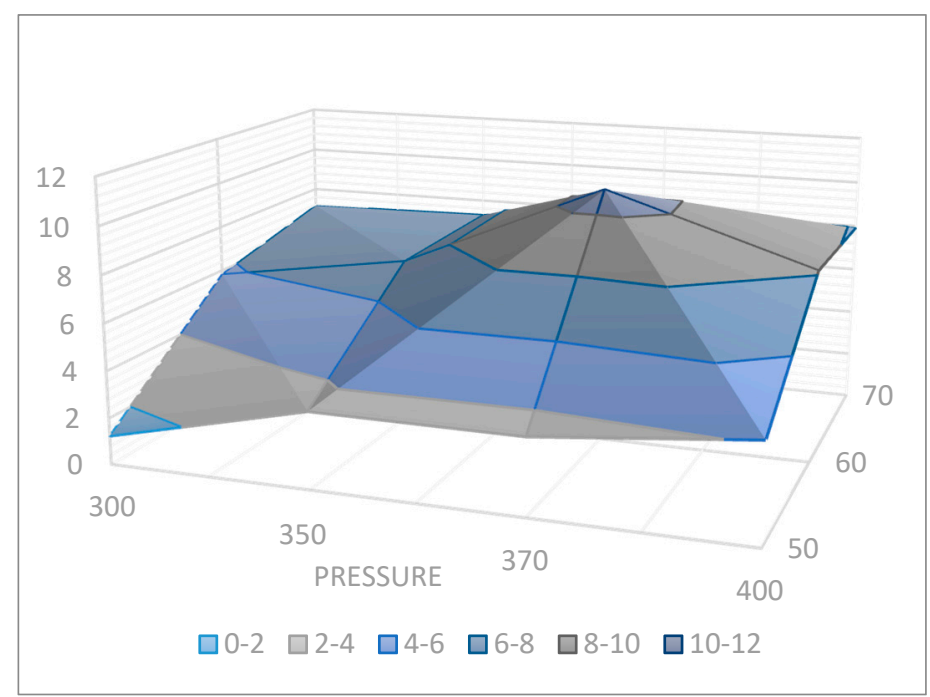

Figure 1. Orthogonal projection representing the extraction yield of target analytes at 300 to 400 Bar and $50-70{ }^{\circ} \mathrm{C}$.

Series studies by HPLC-MS/MS of both samples of rhododendrons ( $R h$. sichotense and Rh. adamsii) were carried out, and the results of studies of the target compounds are presented below (Table 3).

Table 4 summarized all the molecular masses of the target analytes isolated from $\mathrm{SC}-\mathrm{CO}_{2}$ of $R h$. sichotense and Rh. adamsii. Among them, 57 biologically active substances were authenticated $(\mathrm{m} / \mathrm{z}$ values and fragment ions) by comparison with the literature data $[4,5,10-13,15,16,30-53]$. A total of 48 different biologically active components have been identified in the Rh. adamsii $\mathrm{SC}-\mathrm{CO}_{2}$ extracts. A total of 31 different biologically active components have been identified in the Rh. sichotense $\mathrm{SC}-\mathrm{CO}_{2}$ extracts. 
Table 3. Compounds identified from SC-CO $\mathrm{CO}_{2}$ extracts by two varieties of rhododendron: $R h$. sichotense and Rh. adamsii.

\begin{tabular}{|c|c|c|c|c|c|c|c|c|c|c|c|c|c|c|c|c|c|c|c|c|c|c|}
\hline NO. & Identification & Formula & $\begin{array}{l}\text { Calculated } \\
\text { Mass }\end{array}$ & $\begin{array}{c}\text { Rh. } \\
\text { adamsii } \\
\text { (104) }\end{array}$ & $\begin{array}{c}\text { Rh. } \\
\text { adamsii } \\
(\mathbf{1 0 5})\end{array}$ & $\begin{array}{c}\text { Rh. } \\
\text { adamsii } \\
(\mathbf{1 0 9 )}\end{array}$ & $\begin{array}{c}\text { Rh. } \\
\text { adamsii } \\
\text { (110) }\end{array}$ & $\begin{array}{c}\text { Rh. } \\
\text { adamsii } \\
(\mathbf{1 0 8 )}\end{array}$ & $\begin{array}{c}\text { Rh. } \\
\text { adamsii } \\
\text { (112) }\end{array}$ & $\begin{array}{c}\text { Rh. } \\
\text { adamsii } \\
\text { (116) }\end{array}$ & $\begin{array}{c}\text { Rh. } \\
\text { adamsii } \\
\text { (117) }\end{array}$ & $\begin{array}{c}\text { Rh. } \\
\text { adamsii } \\
\text { (118) }\end{array}$ & $\begin{array}{c}\text { Rh. } \\
\text { adamsii } \\
\text { (122) }\end{array}$ & $\begin{array}{c}\text { Rh. } \\
\text { adamsii } \\
\text { (123) }\end{array}$ & $\begin{array}{l}\text { Rh. } \\
\text { sicho } \\
\text { (175) }\end{array}$ & $\begin{array}{l}\text { Rh. } \\
\text { sicho } \\
(176)\end{array}$ & $\begin{array}{l}\text { Rh. } \\
\text { sicho } \\
\text { (177) }\end{array}$ & $\begin{array}{l}\text { Rh. } \\
\text { sicho } \\
\text { (178) }\end{array}$ & $\begin{array}{l}\text { Rh. } \\
\text { sicho } \\
\text { (190) }\end{array}$ & $\begin{array}{l}\text { Rh. } \\
\text { sicho } \\
\text { (192) }\end{array}$ & $\begin{array}{l}\text { Rh. } \\
\text { sicho } \\
\text { (193) }\end{array}$ & References \\
\hline 1 & $\begin{array}{l}\text { Lepalol [5-(3-Furyl)- } \\
\text { 2-methyl-1- } \\
\text { penten-3-ol }\end{array}$ & $\mathrm{C}_{10} \mathrm{H}_{14} \mathrm{O}_{2}$ & 166.217 & & & & & & & & & & & & & & & & & & & {$[31,45,48]$} \\
\hline 2 & $\begin{array}{l}\text { Caffeic acid } \\
\text { [(2E)-3-(3,4- } \\
\text { Dihydroxyphenyl) } \\
\text { acrylic acid] }\end{array}$ & $\mathrm{C}_{9} \mathrm{H}_{8} \mathrm{O}_{4}$ & 180.1574 & & & & & & & & & & & & & & & & & & & $\begin{array}{c}{[10,35,42,43} \\
50]\end{array}$ \\
\hline 3 & $\begin{array}{c}\text { Azelaic acid } \\
\text { (Nonanedioic acid) }\end{array}$ & $\mathrm{C}_{9} \mathrm{H}_{16} \mathrm{O}_{4}$ & 188.2209 & & & & & & & & & & & & & & & & & & & {$[15,16]$} \\
\hline 4 & $\begin{array}{c}\text { Calamenene } \\
\text { [Cis-Calamenene] }\end{array}$ & $\mathrm{C}_{15} \mathrm{H}_{22}$ & 202.3352 & & & & & & & & & & & & & & & & & & & {$[15,16,38]$} \\
\hline 5 & Germacron & $\mathrm{C}_{15} \mathrm{H}_{22} \mathrm{O}$ & 218.3346 & & & & & & & & & & & & & & & & & & & {$[4,5,10,42,44]$} \\
\hline 6 & $\begin{array}{c}\text { Myristic acid } \\
\text { (Tetradecanoic acid; } \\
\text { N-Tetradecanoic } \\
\text { acid) }\end{array}$ & $\mathrm{C}_{14} \mathrm{H}_{28} \mathrm{O}_{2}$ & 228.3709 & & & & & & & & & & & & & & & & & & & {$[15,16]$} \\
\hline 7 & $\begin{array}{l}\text { Pentadecanoic acid } \\
\text { (Pentadecylic acid) }\end{array}$ & $\mathrm{C}_{15} \mathrm{H}_{30} \mathrm{O}_{2}$ & 242.3975 & & & & & & & & & & & & & & & & & & & {$[15,16]$} \\
\hline 8 & Palmitoleic acid & $\mathrm{C}_{16} \mathrm{H}_{30} \mathrm{O}_{2}$ & 254.4082 & & & & & & & & & & & & & & & & & & & {$[15,16]$} \\
\hline 9 & $\begin{array}{l}\text { Cis-cyclopropan- } \\
\text { 9,10-hexadecanoic } \\
\text { acid } \\
\end{array}$ & $\mathrm{C}_{17} \mathrm{H}_{32} \mathrm{O}_{2}$ & 268.4348 & & & & & & & & & & & & & & & & & & & {$[15,16]$} \\
\hline 10 & $\begin{array}{l}\text { Linoleic acid (Linolic } \\
\text { acid; Telfairic acid) }\end{array}$ & $\mathrm{C}_{18} \mathrm{H}_{32} \mathrm{O}_{2}$ & 280.4455 & & & & & & & & & & & & & & & & & & & {$[15,16,49]$} \\
\hline 11 & $\begin{array}{c}\text { Stearic acid } \\
\text { (Octadecanoic acid; } \\
\text { Stearophanic acid) }\end{array}$ & $\mathrm{C}_{18} \mathrm{H}_{36} \mathrm{O}_{2}$ & 284.4772 & & & & & & & & & & & & & & & & & & & {$[15,16]$} \\
\hline 12 & Kaempferol & $\mathrm{C}_{15} \mathrm{H}_{10} \mathrm{O}_{6}$ & 286.2363 & & & & & & & & & & & & & & & & & & & $\begin{array}{l}{[36,39,40,43,} \\
50]\end{array}$ \\
\hline 13 & $\begin{array}{l}\text { Cis-cyclopropan- } \\
\text { 9,10-octadecanoic } \\
\text { acid }\end{array}$ & $\mathrm{C}_{19} \mathrm{H}_{32} \mathrm{O}_{2}$ & 292.4562 & & & & & & & & & & & & & & & & & & & {$[15,16]$} \\
\hline 14 & $\begin{array}{l}\text { Nonadecanoic acid } \\
\text { (N-Nonadecanoic } \\
\text { acid) }\end{array}$ & $\mathrm{C}_{19} \mathrm{H}_{38} \mathrm{O}_{2}$ & 298.5038 & & & & & & & & & & & & & & & & & & & {$[15,16]$} \\
\hline 15 & $\begin{array}{l}\text { Kaempferol 5-methyl } \\
\text { ether }\end{array}$ & $\mathrm{C}_{16} \mathrm{H}_{12} \mathrm{O}_{6}$ & 300.2629 & & & & & & & & & & & & & & & & & & & [39] \\
\hline 16 & Farrerol & $\mathrm{C}_{17} \mathrm{H}_{16} \mathrm{O}_{5}$ & 300.3059 & & & & & & & & & & & & & & & & & & & {$[34,39]$} \\
\hline 17 & Quercetin & $\mathrm{C}_{15} \mathrm{H}_{10} \mathrm{O}_{7}$ & 302.2357 & & & & & & & & & & & & & & & & & & & $\begin{array}{c}{[34,36,39,40} \\
43,50]\end{array}$ \\
\hline 18 & $\begin{array}{c}\text { Dihydroquercetin } \\
\text { (Taxifolin; Taxifoliol) }\end{array}$ & $\mathrm{C}_{15} \mathrm{H}_{12} \mathrm{O}_{7}$ & 304.2516 & & & & & & & & & & & & & & & & & & & {$[35,39,40]$} \\
\hline 19 & $\begin{array}{c}\text { Cannabigerorcinic } \\
\text { acid } \\
\text { (Cannabigerorcinolic } \\
\text { acid; } \\
\text { Cannabiorcogerolic } \\
\text { acid }\end{array}$ & $\mathrm{C}_{18} \mathrm{H}_{24} \mathrm{O}_{4}$ & 304.3808 & & & & & & & & & & & & & & & & & & & {$[15,16]$} \\
\hline
\end{tabular}


Table 3. Cont

\begin{tabular}{|c|c|c|c|c|c|c|c|c|c|c|c|c|c|c|c|c|c|c|c|c|c|c|}
\hline NO. & Identification & Formula & $\begin{array}{l}\text { Calculated } \\
\text { Mass }\end{array}$ & $\begin{array}{c}\text { Rh. } \\
\text { adamsii } \\
(\mathbf{1 0 4})\end{array}$ & $\begin{array}{c}R h . \\
\text { adamsii } \\
(\mathbf{1 0 5})\end{array}$ & $\begin{array}{c}R h . \\
\text { adamsii } \\
\text { (109) }\end{array}$ & $\begin{array}{c}\text { Rh. } \\
\text { adamsii } \\
\text { (110) }\end{array}$ & $\begin{array}{c}\text { Rh. } \\
\text { adamsii } \\
(\mathbf{1 0 8})\end{array}$ & $\begin{array}{c}\text { Rh. } \\
\text { adamsii } \\
(112)\end{array}$ & $\begin{array}{c}\text { Rh. } \\
\text { adamsii } \\
\text { (116) }\end{array}$ & $\begin{array}{c}R h . \\
\text { adamsii } \\
(117)\end{array}$ & $\begin{array}{c}R h . \\
\text { adamsii } \\
(118)\end{array}$ & $\begin{array}{c}R h . \\
\text { adamsii } \\
(\mathbf{1 2 2})\end{array}$ & $\begin{array}{c}R h . \\
\text { adamsii } \\
(\mathbf{1 2 3})\end{array}$ & $\begin{array}{l}\text { Rh. } \\
\text { sicho } \\
(175) \\
\end{array}$ & $\begin{array}{l}\text { Rh. } \\
\text { sicho } \\
\text { (176) }\end{array}$ & $\begin{array}{l}\text { Rh. } \\
\text { sicho } \\
\text { (177) }\end{array}$ & $\begin{array}{l}\text { Rh. } \\
\text { sicho } \\
\text { (178) }\end{array}$ & $\begin{array}{l}\text { Rh. } \\
\text { sicho } \\
\text { (190) }\end{array}$ & $\begin{array}{l}\text { Rh. } \\
\text { sicho } \\
\text { (192) }\end{array}$ & $\begin{array}{l}\text { Rh. } \\
\text { sicho } \\
\text { (193) }\end{array}$ & References \\
\hline 20 & Docosane & $\mathrm{C}_{22} \mathrm{H}_{46}$ & 310.6006 & & & & & & & & & & & & & & & & & & & [48] \\
\hline 21 & $\begin{array}{c}\text { 8-Demethyleucalyptin } \\
\text { [5-Hydroxy-4',7- } \\
\text { dimetoxy-6- } \\
\text { methylflavone; } \\
\text { Pabalate; sodium } \\
\text { salicylate] } \\
\end{array}$ & $\mathrm{C}_{18} \mathrm{H}_{16} \mathrm{O}_{5}$ & 312.3166 & & & & & & & & & & & & & & & & & & & [33] \\
\hline 22 & $\begin{array}{l}\text { Arachic acid } \\
\text { (Arachidic acid; } \\
\text { eicosanoic acid) }\end{array}$ & $\mathrm{C}_{20} \mathrm{H}_{40} \mathrm{O}_{2}$ & 312.5304 & & & & & & & & & & & & & & & & & & & {$[15,16]$} \\
\hline 23 & $\begin{array}{c}\text { Azaleatin } \\
\text { [5-O-Methylquercetin] }\end{array}$ & $\mathrm{C}_{16} \mathrm{H}_{12} \mathrm{O}_{7}$ & 316.2623 & & & & & & & & & & & & & & & & & & & {$[10,39,41,42]$} \\
\hline 24 & Myricetin & $\mathrm{C}_{15} \mathrm{H}_{10} \mathrm{O}_{8}$ & 318.2351 & & & & & & & & & & & & & & & & & & & $\begin{array}{c}{[34,36,39,40,} \\
50]\end{array}$ \\
\hline 25 & $\begin{array}{l}\text { Gossypetin } \\
\text { [Articulatidin; } \\
\text { Equisporol] }\end{array}$ & $\mathrm{C}_{15} \mathrm{H}_{10} \mathrm{O}_{8}$ & 318.2351 & & & & & & & & & & & & & & & & & & & [37] \\
\hline 26 & $\begin{array}{l}\text { Ampelopsin } \\
\text { [Dihydromyricetin; } \\
\text { Ampeloptin] }\end{array}$ & $\mathrm{C}_{15} \mathrm{H}_{12} \mathrm{O}_{8}$ & 320.251 & & & & & & & & & & & & & & & & & & & [39] \\
\hline 27 & $\begin{array}{l}\text { Heneicosanoic acid } \\
\text { (Heneicosylic acid) }\end{array}$ & $\mathrm{C}_{21} \mathrm{H}_{42} \mathrm{O}_{2}$ & 326.557 & & & & & & & & & & & & & & & & & & & {$[15,16]$} \\
\hline 28 & $\begin{array}{c}\text { Myricetin 5-Methyl } \\
\text { ether } \\
\text { [5-O-Methylmyricetin] }\end{array}$ & $\mathrm{C}_{16} \mathrm{H}_{12} \mathrm{O}_{8}$ & 332.2617 & & & & & & & & & & & & & & & & & & & [39] \\
\hline 29 & $\begin{array}{l}\text { Esculin [Aesculin; } \\
\text { Esculoside; } \\
\text { Polichrome] }\end{array}$ & $\mathrm{C}_{15} \mathrm{H}_{16} \mathrm{O}_{9}$ & 340.2821 & & & & & & & & & & & & & & & & & & & {$[33,41]$} \\
\hline 30 & $\begin{array}{c}\text { Behenic acid } \\
\text { (Docosanoic acid) } \\
\end{array}$ & $\mathrm{C}_{22} \mathrm{H}_{44} \mathrm{O}_{2}$ & 340.5836 & & & & & & & & & & & & & & & & & & & {$[15,16]$} \\
\hline 31 & $\begin{array}{c}\text { Pentacosane } \\
\text { (N-Pentacosane) }\end{array}$ & $\mathrm{C}_{25} \mathrm{H}_{52}$ & 352.6854 & & & & & & & & & & & & & & & & & & & [48] \\
\hline 32 & Chlorogenic acid & $\mathrm{C}_{16} \mathrm{H}_{18} \mathrm{O}_{9}$ & 354.3087 & & & & & & & & & & & & & & & & & & & {$[10,35,42]$} \\
\hline 33 & $\begin{array}{l}\text { Scopolin [Scopoloside; } \\
\text { Scopoletin-7-glucoside; } \\
\text { Murrayin] }\end{array}$ & $\mathrm{C}_{16} \mathrm{H}_{18} \mathrm{O}_{9}$ & 354.3087 & & & & & & & & & & & & & & & & & & & [41] \\
\hline 34 & $\begin{array}{c}\text { Tricosanoic acid } \\
\text { (N-Tricosanoic acid) }\end{array}$ & $\mathrm{C}_{23} \mathrm{H}_{46} \mathrm{O}_{2}$ & 354.6101 & & & & & & & & & & & & & & & & & & & {$[15,16]$} \\
\hline 35 & $\begin{array}{c}\text { Lignoceric acid } \\
\text { (Tetracosanoic acid) }\end{array}$ & $\mathrm{C}_{24} \mathrm{H}_{48} \mathrm{O}_{2}$ & 368.6367 & & & & & & & & & & & & & & & & & & & {$[15,16]$} \\
\hline 36 & $\begin{array}{c}\text { Fraxin } \\
\text { (Fraxetin-8-O-glucoside) }\end{array}$ & $\mathrm{C}_{16} \mathrm{H}_{18} \mathrm{O}_{10}$ & 370.3081 & & & & & & & & & & & & & & & & & & & [33] \\
\hline 37 & Daurichromenic acid & $\mathrm{C}_{23} \mathrm{H}_{30} \mathrm{O}_{4}$ & 370.4819 & & & & & & & & & & & & & & & & & & & {$[15,16]$} \\
\hline 38 & $\begin{array}{c}\text { Pentacosanoic acid } \\
\text { (N-Pentacosanoic acid) }\end{array}$ & $\mathrm{C}_{25} \mathrm{H}_{50} \mathrm{O}_{2}$ & 382.6633 & & & & & & & & & & & & & & & & & & & {$[15,16]$} \\
\hline 39 & $\begin{array}{l}\text { Fraxetin-7-O-beta- } \\
\text { glucuronide }\end{array}$ & $\mathrm{C}_{16} \mathrm{H}_{16} \mathrm{O}_{11}$ & 384.2916 & & & & & & & & & & & & & & & & & & & [41] \\
\hline 40 & $\begin{array}{l}\text { Beta-Sitosterin } \\
\text { [Beta-Sitosterol] }\end{array}$ & $\mathrm{C}_{29} \mathrm{H}_{50} \mathrm{O}$ & 414.7067 & & & & & & & & & & & & & & & & & & & {$[10,30,42]$} \\
\hline
\end{tabular}


Table 3. Cont.

\begin{tabular}{|c|c|c|c|c|c|c|c|c|c|c|c|c|c|c|c|c|c|c|c|c|c|c|}
\hline No. & Identification & Formula & $\begin{array}{l}\text { Calculated } \\
\text { Mass }\end{array}$ & $\begin{array}{c}R h . \\
\text { adamsii } \\
(\mathbf{1 0 4 )}\end{array}$ & $\begin{array}{c}R h . \\
\text { adamsii } \\
(\mathbf{1 0 5})\end{array}$ & $\begin{array}{c}\text { Rh. } \\
\text { adamsii } \\
\text { (109) }\end{array}$ & $\begin{array}{c}R h . \\
\text { adamsii } \\
(110)\end{array}$ & $\begin{array}{c}R h . \\
\text { adamsii } \\
(\mathbf{1 0 8 )}\end{array}$ & $\begin{array}{c}R h . \\
\text { adamsii } \\
(\mathbf{1 1 2})\end{array}$ & $\begin{array}{c}R h . \\
\text { adamsii } \\
(116)\end{array}$ & $\begin{array}{c}R h . \\
\text { adamsii } \\
(117)\end{array}$ & $\begin{array}{c}R h . \\
\text { adamsii } \\
(118)\end{array}$ & $\begin{array}{c}R h . \\
\text { adamsii } \\
\text { (122) }\end{array}$ & $\begin{array}{c}R h . \\
\text { adamsii } \\
\text { (123) }\end{array}$ & $\begin{array}{l}\text { Rh. } \\
\text { sicho } \\
\text { (175) }\end{array}$ & $\begin{array}{l}\text { Rh. } \\
\text { sicho } \\
(176)\end{array}$ & $\begin{array}{l}\text { Rh. } \\
\text { sicho } \\
\text { (177) }\end{array}$ & $\begin{array}{l}\text { Rh. } \\
\text { sicho } \\
(178) \\
\end{array}$ & $\begin{array}{l}\text { Rh. } \\
\text { sicho } \\
(190)\end{array}$ & $\begin{array}{l}\text { Rh. } \\
\text { sicho } \\
\text { (192) } \\
\end{array}$ & $\begin{array}{l}\text { Rh. } \\
\text { sicho } \\
(193) \\
\end{array}$ & References \\
\hline 41 & $\begin{array}{l}\text { Cyanidin-3-alpfa-L- } \\
\text { arabinoside }\end{array}$ & $\mathrm{C}_{20} \mathrm{H}_{19} \mathrm{O}_{10}$ & 419.3589 & & & & & & & & & & & & & & & & & & & {$[10,42]$} \\
\hline 42 & $\begin{array}{l}\text { Montanic acid } \\
\text { (Amyrin; } \\
\text { Beta-Amyrenol) }\end{array}$ & $\mathrm{C}_{28} \mathrm{H}_{56} \mathrm{O}_{2}$ & 424.743 & & & & & & & & & & & & & & & & & & & {$[15,16]$} \\
\hline 43 & $\begin{array}{l}\text { Alpha-Amyrin } \\
\text { [Viminalol] }\end{array}$ & $\mathrm{C}_{30} \mathrm{H}_{50} \mathrm{O}$ & 426.7174 & & & & & & & & & & & & & & & & & & & [30] \\
\hline 44 & $\begin{array}{c}\text { Lupeol [Fagarasterol; } \\
\text { Clerodol; Monogynol } \\
\text { B; Lupenol] }\end{array}$ & $\mathrm{C}_{30} \mathrm{H}_{50} \mathrm{O}$ & 426.7174 & & & & & & & & & & & & & & & & & & & [30] \\
\hline 45 & $\begin{array}{l}\text { Dihydroquercetin-3- } \\
\text { arabinofuranoside }\end{array}$ & $\mathrm{C}_{20} \mathrm{H}_{16} \mathrm{O}_{11}$ & 432.3344 & & & & & & & & & & & & & & & & & & & {$[10,42]$} \\
\hline 46 & $\begin{array}{l}\text { Afzelin [ Kaempferol- } \\
\text { 3-Rhamnoside; } \\
\text { Kaempferin] }\end{array}$ & $\mathrm{C}_{21} \mathrm{H}_{20} \mathrm{O}_{10}$ & 432.3775 & & & & & & & & & & & & & & & & & & & {$[39,40]$} \\
\hline 47 & $\begin{array}{l}\text { Quercetin-3-O-beta- } \\
\text { xyloside (Reynoutrin; } \\
\text { Quercetin 3-O-Beta--D- } \\
\text { Xylopyranoside) }\end{array}$ & $\mathrm{C}_{20} \mathrm{H}_{17} \mathrm{O}_{11}$ & 433.3424 & & & & & & & & & & & & & & & & & & & [34] \\
\hline 48 & $\begin{array}{l}\text { Avicularin (Quercetin } \\
\text { 3-Alpha-L- } \\
\text { Arabinofuranoside; } \\
\text { Avicularoside) }\end{array}$ & $\mathrm{C}_{20} \mathrm{H}_{18} \mathrm{O}_{11}$ & 434.3503 & & & & & & & & & & & & & & & & & & & $\begin{array}{c}{[10,33,39,40,} \\
42]\end{array}$ \\
\hline 49 & $\begin{array}{c}\text { Pentoside } \\
\text { dihydroquercetin }\end{array}$ & & 436 & & & & & & & & & & & & & & & & & & & [40] \\
\hline 50 & $\begin{array}{l}\text { Erithrodiol } \\
\text { [3-beta-Erytrodiol] }\end{array}$ & $\mathrm{C}_{30} \mathrm{H}_{50} \mathrm{O}_{2}$ & 442.7168 & & & & & & & & & & & & & & & & & & & [30] \\
\hline 51 & Uvaol & $\mathrm{C}_{30} \mathrm{H}_{50} \mathrm{O}_{2}$ & 442.7168 & & & & & & & & & & & & & & & & & & & [30] \\
\hline 52 & $\begin{array}{l}\text { Quercitrin [Quercetin } \\
\text { 3-L- Rhamnoside; } \\
\text { Quercetrin] }\end{array}$ & $\mathrm{C}_{21} \mathrm{H}_{20} \mathrm{O}_{11}$ & 448.3769 & & & & & & & & & & & & & & & & & & & {$[33,39,46]$} \\
\hline 53 & Catechin-7-O-glucoside & $\mathrm{C}_{21} \mathrm{H}_{24} \mathrm{O}_{11}$ & 452.4087 & & & & & & & & & & & & & & & & & & & [34] \\
\hline 54 & Micromeric acid & $\mathrm{C}_{30} \mathrm{H}_{46} \mathrm{O}_{3}$ & 454.6844 & & & & & & & & & & & & & & & & & & & [30] \\
\hline 55 & $\begin{array}{l}\text { Hyperoside (Quercetin } \\
\text { 3-O-galactoside; } \\
\text { Hyperin) }\end{array}$ & $\mathrm{C}_{21} \mathrm{H}_{20} \mathrm{O}_{12}$ & 464.3763 & & & & & & & & & & & & & & & & & & & $\begin{array}{c}{[10,33,34,39-} \\
42]\end{array}$ \\
\hline 56 & $\begin{array}{c}\text { Quercetin } \\
\text { 3-O-glucoside [ } \\
\text { Isoquercitrin] }\end{array}$ & $\mathrm{C}_{21} \mathrm{H}_{20} \mathrm{O}_{12}$ & 464.3763 & & & & & & & & & & & & & & & & & & & {$[33,46]$} \\
\hline 57 & $\begin{array}{l}\text { Alpha.--Tocopherol-Beta- } \\
\text { D-Mannoside } \\
\text { [Dihydro-2H-Chromen- } \\
\text { 6-YI Hexofuranoside] }\end{array}$ & $\mathrm{C}_{35} \mathrm{H}_{60} \mathrm{O}_{7}$ & 592.8467 & & & & & & & & & & & & & & & & & & & [48] \\
\hline
\end{tabular}

Colors are added for readability to avoid confusing columns. Green shades Rh. adamsii. blue Rh. sichotense. 
Table 4. Components identified from the $\mathrm{SC}-\mathrm{CO}_{2}$ extracts of $\mathrm{Rh}$. sichotense and Rh. adamsii.

\begin{tabular}{|c|c|c|c|c|c|c|c|c|c|c|}
\hline No. & Identification & Formula & $\begin{array}{l}\text { Calculated } \\
\text { Mass }\end{array}$ & $\begin{array}{l}\text { Observed Mass } \\
{[\mathrm{M}-\mathrm{H}]^{-}}\end{array}$ & $\begin{array}{c}\text { Observed Mass } \\
{[\mathrm{M}+\mathrm{H}]^{+}}\end{array}$ & $\begin{array}{c}\text { Observed Mass } \\
{[\mathrm{M}+\mathrm{Na}]^{+}}\end{array}$ & $\begin{array}{l}\text { MS/MS Stage } 2 \\
\text { Fragmentation }\end{array}$ & $\begin{array}{l}\text { MS/MS Stage } 3 \\
\text { Fragmentation }\end{array}$ & $\begin{array}{c}\text { MS/MS Stage } 4 \\
\text { Fragmentation }\end{array}$ & $\begin{array}{c}\text { Species of } \\
\text { Rhododendron }\end{array}$ \\
\hline 1 & $\begin{array}{c}\text { Lepalol [5-(3-Furyl) } \\
\text {-2-methyl-1-penten-3-ol }\end{array}$ & $\mathrm{C}_{10} \mathrm{H}_{14} \mathrm{O}_{2}$ & 166.217 & 165.06 & & & 147.01 & & & Rh. adamsii \\
\hline 2 & $\begin{array}{l}\text { Caffeic acid [(2E)-3- } \\
\text { (3,4-Dihydroxyphenyl) } \\
\text { acrylic acid] }\end{array}$ & $\mathrm{C}_{9} \mathrm{H}_{8} \mathrm{O}_{4}$ & 180.1574 & & 181.08 & & $163.03 ; 135.11$ & & & Rh. adamsii \\
\hline 3 & $\begin{array}{c}\text { Azelaic acid } \\
\text { [Nonanedioic acid] }\end{array}$ & $\mathrm{C}_{9} \mathrm{H}_{16} \mathrm{O}_{4}$ & 188.2209 & & & 210.09 & 192.12 & 175.06; 136.12 & & Rh. adamsii \\
\hline 4 & $\begin{array}{c}\text { Calamenene } \\
\text { [Cis-Calamenene] }\end{array}$ & $\mathrm{C}_{15} \mathrm{H}_{22}$ & 202.3352 & & 203.09 & & 147.05 & 119.06 & & Rh. sichotense \\
\hline 5 & Germacron & $\mathrm{C}_{15} \mathrm{H}_{22} \mathrm{O}$ & 218.3346 & & 219.06 & & $201.07 ; 149.07$ & 159.07 & & Rh. adamsii \\
\hline 6 & $\begin{array}{c}\text { Myristic acid } \\
\text { (Tetradecanoic acid; } \\
N \text {-Tetradecanoic acid) }\end{array}$ & $\mathrm{C}_{14} \mathrm{H}_{28} \mathrm{O}_{2}$ & 228.3709 & & & 251.09 & 150.48 & 149.08 & & Rh. adamsii \\
\hline 7 & $\begin{array}{l}\text { Pentadecanoic acid } \\
\text { (Pentadecylic acid) }\end{array}$ & $\mathrm{C}_{15} \mathrm{H}_{30} \mathrm{O}_{2}$ & 242.3975 & & 243.06 & & $201.01 ; 137.05$ & 181.05; 135.04 & & Rh. adamsii \\
\hline 8 & Palmitoleic acid & $\mathrm{C}_{16} \mathrm{H}_{30} \mathrm{O}_{2}$ & 254.4082 & & & 277.09 & $275.04 ; 207.05$ & 256.99 & 157.11 & Rh. adamsii \\
\hline 9 & $\begin{array}{l}\text { Cis-cyclopropan-9,10- } \\
\text { hexadecanoic acid }\end{array}$ & $\mathrm{C}_{17} \mathrm{H}_{32} \mathrm{O}_{2}$ & 268.4348 & & 269.02 & & $185.97 ; 121.08$ & 176.96 & 154.98 & Rh. adamsii \\
\hline 10 & $\begin{array}{c}\text { Linoleic acid [Linolic acid; } \\
\text { Telfairic acid] }\end{array}$ & $\mathrm{C}_{18} \mathrm{H}_{32} \mathrm{O}_{2}$ & 280.4455 & & & 303.06 & $285.05 ; 163.00$ & $180.95 ; 135.06$ & 162.99 & Rh. adamsii \\
\hline 11 & $\begin{array}{c}\text { Stearic acid } \\
\text { [Octadecanoic acid; } \\
\text { Stearophanic acid] }\end{array}$ & $\mathrm{C}_{18} \mathrm{H}_{36} \mathrm{O}_{2}$ & 284.4772 & & 285.07 & & $\begin{array}{l}284.18 ; 229.07 \\
\quad 163.02\end{array}$ & 180.90; 135.05 & 163.03 & $\begin{array}{l}\text { Rh. adamsii; } \\
\text { Rh. sichotense }\end{array}$ \\
\hline 12 & $\begin{array}{c}\text { Kaempferol [3,5,7- } \\
\text { Trihydroxy-2-(4-hydro- } \\
\text { xyphenyl)-4-H-chromen- } \\
\text { 4-one] }\end{array}$ & $\mathrm{C}_{15} \mathrm{H}_{10} \mathrm{O}_{6}$ & 286.2363 & & 287.00 & & $\begin{array}{l}286.24 ; 204.96 \\
163.02\end{array}$ & 181.02 & 162.88 & Rh. adamsii \\
\hline 13 & $\begin{array}{l}\text { Cis-cyclopropan-9,10- } \\
\text { octadecanoic acid }\end{array}$ & $\mathrm{C}_{19} \mathrm{H}_{32} \mathrm{O}_{2}$ & 292.4562 & & 293.05 & & 274.98 & $256.99 ; 162.98$ & 201.03 & Rh. adamsii \\
\hline 14 & $\begin{array}{c}\text { Nonadecanoic acid } \\
\text { [N-Nonadecanoic acid] }\end{array}$ & $\mathrm{C}_{19} \mathrm{H}_{38} \mathrm{O}_{2}$ & 298.5038 & & 300.09 & & 243.04 & 201.02 & & $\begin{array}{l}\text { Rh. adamsii; } \\
\text { Rh. sichotense }\end{array}$ \\
\hline 15 & $\begin{array}{l}\text { Kaempferol 5-methyl } \\
\text { ether }\end{array}$ & $\mathrm{C}_{16} \mathrm{H}_{12} \mathrm{O}_{6}$ & 300.2629 & & 300.98 & & $283.01 ; 177.01$ & 264.98 & 200.98 & $\begin{array}{l}\text { Rh. adamsii; } \\
\text { Rh. sichotense }\end{array}$ \\
\hline
\end{tabular}


Table 4. Cont

\begin{tabular}{|c|c|c|c|c|c|c|c|c|c|c|}
\hline No. & Identification & Formula & $\begin{array}{l}\text { Calculated } \\
\text { Mass }\end{array}$ & $\begin{array}{l}\text { Observed Mass } \\
{[\mathbf{M}-\mathbf{H}]^{-}}\end{array}$ & $\begin{array}{l}\text { Observed Mass } \\
\qquad[\mathrm{M}+\mathrm{H}]^{+}\end{array}$ & $\begin{array}{l}\text { Observed Mass } \\
{[\mathrm{M}+\mathrm{Na}]^{+}}\end{array}$ & $\begin{array}{l}\text { MS/MS Stage } 2 \\
\text { Fragmentation }\end{array}$ & $\begin{array}{l}\text { MS/MS Stage } 3 \\
\text { Fragmentation }\end{array}$ & $\begin{array}{l}\text { MS/MS Stage } 4 \\
\text { Fragmentation }\end{array}$ & $\begin{array}{c}\text { Species of } \\
\text { Rhododendron }\end{array}$ \\
\hline 16 & $\begin{array}{l}\text { Farrerol [5,7-Dihydroxy- } \\
\text { 2-(4-hydroxyphenyl)-6,8- } \\
\text { dimethylchroman-4-one] }\end{array}$ & $\mathrm{C}_{17} \mathrm{H}_{16} \mathrm{O}_{5}$ & 300.3059 & & 301.05 & & 283.04 & $241.01 ; 162.96$ & & $\begin{array}{l}\text { Rh. adamsii; } \\
\text { Rh. sichotense }\end{array}$ \\
\hline 17 & $\begin{array}{c}\text { Quercetin [2-(3,4- } \\
\text { Dihydroxyphenyl)-3,5,7- } \\
\text { trihy- droxy-4- } H- \\
\text { chromen-4-one] }\end{array}$ & $\mathrm{C}_{15} \mathrm{H}_{12} \mathrm{O}_{7}$ & 302.2357 & 301.09 & 303.08 & & $285.01 ; 163.02$ & $180.97 ; 145.00$ & 162.98 & $\begin{array}{l}\text { Rh. adamsii; } \\
\text { Rh. sichotense }\end{array}$ \\
\hline 18 & $\begin{array}{c}\text { Dihydroquercetin } \\
\text { [Taxifolin; Taxifoliol] }\end{array}$ & $\mathrm{C}_{15} \mathrm{H}_{12} \mathrm{O}_{7}$ & 304.2516 & 303.09 & & & 285.04 & $\begin{array}{l}266.96 ; 241.09 \\
215.05 ; 135.05\end{array}$ & 171.02 & Rh. adamsii \\
\hline 19 & $\begin{array}{l}\text { Cannabigerorcinic acid } \\
\text { [Cannabigerorcinolic acid; } \\
\text { Cannabiorcogerolic acid] }\end{array}$ & $\mathrm{C}_{18} \mathrm{H}_{24} \mathrm{O}_{4}$ & 304.3808 & 303.08 & & & 285.05 & $241.07 ; 159.07$ & 159.01 & Rh. adamsii \\
\hline 20 & Docosane & $\mathrm{C}_{22} \mathrm{H}_{46}$ & 310.6006 & & 311.10 & & $293.06 ; 167.01$ & 259.03 & $240.97 ; 162.96$ & Rh. adamsii \\
\hline 21 & $\begin{array}{c}\text { 8-Demethyleucalyptin } \\
\text { [5-Hydroxy-4',7-dimetoxy- } \\
\text { 6-methylflavone; } \\
\text { Pabalate; Sodium } \\
\text { salicylate] }\end{array}$ & $\mathrm{C}_{18} \mathrm{H}_{16} \mathrm{O}_{5}$ & 312.3166 & 311.14 & & & 311.10; 182.99 & & & Rh. adamsii \\
\hline 22 & $\begin{array}{l}\text { Arachic acid [Arachidic } \\
\text { acid; eicosanoic acid] }\end{array}$ & $\mathrm{C}_{20} \mathrm{H}_{40} \mathrm{O}_{2}$ & 312.5304 & 311.14 & & 335.04 & 303.06; 195.01 & $\begin{array}{c}284.99 ; 238.14 \\
163.00\end{array}$ & $180.89 ; 135.14$ & Rh. adamsii \\
\hline 23 & $\begin{array}{c}\text { Azaleatin } \\
\text { [5-O-Methylquercetin] }\end{array}$ & $\mathrm{C}_{16} \mathrm{H}_{12} \mathrm{O}_{7}$ & 316.2623 & 315.08 & & & $297.01 ; 167.04$ & $235.04 ; 149.00$ & & Rh. adamsii \\
\hline 24 & $\begin{array}{c}\text { Myricetin } \\
\text { [3,5,7-Trihydroxy- } \\
\text { 2-(3,4,5-Trihydroxyphenyl)- } \\
\text { 4H-Chromen-4-One] }\end{array}$ & $\mathrm{C}_{15} \mathrm{H}_{10} \mathrm{O}_{8}$ & 318.2351 & 317.08 & & & $299.01 ; 241.01$ & $240.06 ; 197.09$ & $238.99 ; 197.04$ & $\begin{array}{l}\text { Rh. adamsii; } \\
\text { Rh. sichotense }\end{array}$ \\
\hline 25 & $\begin{array}{l}\text { Gossypetin [Articulatidin; } \\
\text { Equisporol] }\end{array}$ & $\mathrm{C}_{15} \mathrm{H}_{10} \mathrm{O}_{8}$ & 318.2351 & & 319.07 & & $287.09 ; 176.98$ & 146.99 & & Rh. adamsii \\
\hline 26 & $\begin{array}{c}\text { Ampelopsin } \\
\text { [Dihydromyricetin; } \\
\text { Ampeloptin] }\end{array}$ & $\mathrm{C}_{15} \mathrm{H}_{12} \mathrm{O}_{8}$ & 320.251 & 319.08 & & & $317.01 ; 275.09$ & $257.12 ; 217.11$ & & Rh. adamsii \\
\hline 27 & $\begin{array}{l}\text { Heneicosanoic acid } \\
\text { [Heneicosylic acid] }\end{array}$ & $\mathrm{C}_{21} \mathrm{H}_{42} \mathrm{O}_{2}$ & 326.557 & 325.11 & 327.08 & & $\begin{array}{l}\text { 271.01; } 217.03 ; \\
177.06\end{array}$ & 149.10 & & $\begin{array}{l}\text { Rh. adamsii; } \\
\text { Rh. sichotense }\end{array}$ \\
\hline
\end{tabular}


Table 4. Cont.

\begin{tabular}{|c|c|c|c|c|c|c|c|c|c|c|}
\hline No. & Identification & Formula & $\begin{array}{l}\text { Calculated } \\
\text { Mass }\end{array}$ & $\begin{array}{l}\text { Observed Mass } \\
{[\mathrm{M}-\mathrm{H}]^{-}}\end{array}$ & $\begin{array}{l}\text { Observed Mass } \\
{[\mathrm{M}+\mathrm{H}]^{+}}\end{array}$ & $\begin{array}{c}\text { Observed Mass } \\
{[\mathrm{M}+\mathrm{Na}]^{+}}\end{array}$ & $\begin{array}{l}\text { MS/MS Stage } 2 \\
\text { Fragmentation }\end{array}$ & $\begin{array}{l}\text { MS/MS Stage } 3 \\
\text { Fragmentation }\end{array}$ & $\begin{array}{c}\text { MS/MS Stage } 4 \\
\text { Fragmentation }\end{array}$ & $\begin{array}{c}\text { Species of } \\
\text { Rhododendron }\end{array}$ \\
\hline 28 & $\begin{array}{l}\text { Myricetin 5-Methyl ether } \\
\text { [5-O-Methylmyricetin] }\end{array}$ & $\mathrm{C}_{16} \mathrm{H}_{12} \mathrm{O}_{8}$ & 332.2617 & 331.03 & & & 168.94 & 149.96 & & Rh. sichotense \\
\hline 29 & $\begin{array}{c}\text { Esculin [Aesculin; } \\
\text { Esculoside; Polichrome] }\end{array}$ & $\mathrm{C}_{15} \mathrm{H}_{16} \mathrm{O}_{9}$ & 340.2821 & & 341.09 & & $\begin{array}{c}\text { 281.01; 217.11; } \\
151.06\end{array}$ & 174.96 & & $\begin{array}{l}\text { Rh. adamsii; } \\
\text { Rh. sichotense }\end{array}$ \\
\hline 30 & $\begin{array}{c}\text { Behenic acid } \\
\text { [Docosanoic acid] }\end{array}$ & $\mathrm{C}_{22} \mathrm{H}_{44} \mathrm{O}_{2}$ & 340.5836 & & 341.05 & & $\begin{array}{c}\text { 323.10; } 243.11 \\
\text { 177.04 }\end{array}$ & 159.05 & & $\begin{array}{l}\text { Rh. adamsii; } \\
\text { Rh. sichotense }\end{array}$ \\
\hline 31 & $\begin{array}{c}\text { Pentacosane } \\
\text { (N-Pentacosane) }\end{array}$ & $\mathrm{C}_{25} \mathrm{H}_{52}$ & 352.6854 & & 353.12 & & $270.97 ; 162.97$ & 180.93 & 162.96 & Rh. sichotense \\
\hline 32 & Chlorogenic acid & $\mathrm{C}_{16} \mathrm{H}_{18} \mathrm{O}_{9}$ & 354.3087 & & 355.09 & & $287.05 ; 164.02$ & 180.95 & 163.03 & $\begin{array}{l}\text { Rh. adamsii; } \\
\text { Rh. sichotense }\end{array}$ \\
\hline 33 & $\begin{array}{c}\text { Scopolin [Scopoloside; } \\
\text { Scopoletin-7-glucoside; } \\
\text { Murrayin] }\end{array}$ & $\mathrm{C}_{16} \mathrm{H}_{18} \mathrm{O}_{9}$ & 354.3087 & & 355.02 & & 323.00 & $303.96 ; 184.89$ & 162.86 & $\begin{array}{l}\text { Rh. adamsii; } \\
\text { Rh. sichotense }\end{array}$ \\
\hline 34 & $\begin{array}{c}\text { Tricosanoic acid } \\
{[N-\text { Tricosanoic acid] }}\end{array}$ & $\mathrm{C}_{23} \mathrm{H}_{46} \mathrm{O}_{2}$ & 354.6101 & & 355.08 & & $322.96 ; 163.00$ & 180.96 & 162.96 & Rh. adamsii \\
\hline 35 & $\begin{array}{c}\text { Lignoceric acid } \\
\text { [Tetracosanoic acid] }\end{array}$ & $\mathrm{C}_{24} \mathrm{H}_{48} \mathrm{O}_{2}$ & 368.6367 & 367.12 & 369.08 & & $\begin{array}{l}351.08 ; 285.02 \\
218.92 ; 162.98\end{array}$ & 163.02 & 144.97 & $\begin{array}{l}\text { Rh. adamsii; } \\
\text { Rh. sichotense }\end{array}$ \\
\hline 36 & $\begin{array}{c}\text { Fraxin } \\
\text { (Fraxetin-8-O-glucoside) }\end{array}$ & $\mathrm{C}_{16} \mathrm{H}_{18} \mathrm{O}_{10}$ & 370.3081 & & 371.08 & & 338.99 & $320.96 ; 177.03$ & 224.96 & $\begin{array}{l}\text { Rh. adamsii; } \\
\text { Rh. sichotense }\end{array}$ \\
\hline 37 & Daurichromenic acid & $\mathrm{C}_{23} \mathrm{H}_{30} \mathrm{O}_{4}$ & 370.4819 & & 371.09 & & $\begin{array}{l}352.98 ; 287.08 \\
235.08 ; 179.02\end{array}$ & $\begin{array}{c}231.04 ; 205.05 \\
162.99\end{array}$ & $180.93 ; 144.97$ & $\begin{array}{l}\text { Rh. adamsii; } \\
\text { Rh. sichotense }\end{array}$ \\
\hline 38 & $\begin{array}{c}\text { Pentacosanoic acid } \\
\text { [N-Pentacosanoic acid] }\end{array}$ & $\mathrm{C}_{25} \mathrm{H}_{50} \mathrm{O}_{2}$ & 382.6633 & & 383.07 & 405.08 & $351.04 ; 287.99$ & 229.04 & 211.03 & $\begin{array}{l}\text { Rh. adamsii; } \\
\text { Rh. sichotense }\end{array}$ \\
\hline 39 & $\begin{array}{l}\text { Fraxetin-7-O-beta- } \\
\text { glucuronide }\end{array}$ & $\mathrm{C}_{16} \mathrm{H}_{16} \mathrm{O}_{11}$ & 384.2916 & 383.09 & & & $365.09 ; 190.96$ & $266.97 ; 215.02$ & 170.97 & $\begin{array}{l}\text { Rh. adamsii; } \\
\text { Rh. sichotense }\end{array}$ \\
\hline 40 & $\begin{array}{l}\text { Beta-Sitosterin } \\
\text { [Beta-Sitosterol] }\end{array}$ & $\mathrm{C}_{29} \mathrm{H}_{50} \mathrm{O}$ & 414.7067 & & 415.04 & & 384.02 & 369.01 & 338.00 & $\begin{array}{l}\text { Rh. adamsii; } \\
\text { Rh. sichotense }\end{array}$ \\
\hline 41 & $\begin{array}{l}\text { Cyanidin-3-alpfa-L- } \\
\text { arabinoside }\end{array}$ & $\mathrm{C}_{20} \mathrm{H}_{19} \mathrm{O}_{10}$ & 419.3589 & 418.51 & & & $\begin{array}{c}\text { 399.05; 319.02; } \\
194.99\end{array}$ & 381.068162 .02 & $337.02 ; 253.08$ & Rh. adamsii \\
\hline 42 & $\begin{array}{c}\text { Montanic acid } \\
\text { [Octacosanoic acid] }\end{array}$ & $\mathrm{C}_{28} \mathrm{H}_{56} \mathrm{O}_{2}$ & 424.743 & & 425.02 & & 407.00 & $\begin{array}{l}389.00 ; 348.98 \\
298.99 ; 240.97\end{array}$ & $\begin{array}{l}\text { 333.00; } 280.97 \\
173.02\end{array}$ & $\begin{array}{l}\text { Rh. adamsii; } \\
\text { Rh. sichotense }\end{array}$ \\
\hline 43 & $\begin{array}{l}\text { Alpha-Amyrin } \\
\text { [Viminalol] }\end{array}$ & $\mathrm{C}_{30} \mathrm{H}_{50} \mathrm{O}$ & 426.7174 & & 427.05 & & $\begin{array}{c}\text { 408.27; 308.99; } \\
202.91\end{array}$ & $389.02 ; 309.01$ & $\begin{array}{c}373.08 ; 229.10 \\
142.80\end{array}$ & $\begin{array}{l}\text { Rh. adamsii; } \\
\text { Rh. sichotense }\end{array}$ \\
\hline
\end{tabular}


Table 4. Cont

\begin{tabular}{|c|c|c|c|c|c|c|c|c|c|c|}
\hline No. & Identification & Formula & $\begin{array}{l}\text { Calculated } \\
\text { Mass }\end{array}$ & $\begin{array}{l}\text { Observed Mass } \\
{[\mathrm{M}-\mathrm{H}]^{-}}\end{array}$ & $\begin{array}{l}\text { Observed Mass } \\
{[\mathrm{M}+\mathrm{H}]^{+}}\end{array}$ & $\begin{array}{c}\text { Observed Mass } \\
{[\mathrm{M}+\mathrm{Na}]^{+}}\end{array}$ & $\begin{array}{c}\text { MS/MS Stage } 2 \\
\text { Fragmentation }\end{array}$ & $\begin{array}{c}\text { MS/MS Stage } 3 \\
\text { Fragmentation }\end{array}$ & $\begin{array}{c}\text { MS/MS Stage } 4 \\
\text { Fragmentation }\end{array}$ & $\begin{array}{c}\text { Species of } \\
\text { Rhododendron }\end{array}$ \\
\hline 44 & $\begin{array}{c}\text { Lupeol [Fagarasterol; } \\
\text { Clerodol; Monogynol B; } \\
\text { Lupenol] }\end{array}$ & $\mathrm{C}_{30} \mathrm{H}_{50} \mathrm{O}$ & 426.7174 & & 427.04 & & $409.01 ; 202.99$ & $389.02 ; 247.99$ & $370.96 ; 264.80$ & Rh. adamsii \\
\hline 45 & $\begin{array}{c}\text { Dihydroquercetin } \\
\text { 3-arabinofuranoside }\end{array}$ & $\mathrm{C}_{20} \mathrm{H}_{16} \mathrm{O}_{11}$ & 432.3344 & & 433.97 & & 352.95 & $323.53 ; 271.96$ & $241.95 ; 181.87$ & Rh. adamsii \\
\hline 46 & $\begin{array}{c}\text { Afzelin [ } \\
\text { Kaempferol-3-Rhamnoside; } \\
\text { Kaempferin] }\end{array}$ & $\mathrm{C}_{21} \mathrm{H}_{20} \mathrm{O}_{10}$ & 432.3775 & 431.04 & & & $\begin{array}{l}\text { 413.00; } 372.98 \\
216.94\end{array}$ & $354.95 ; 167.01$ & $336.98 ; 148.91$ & Rh. sichotense \\
\hline 47 & $\begin{array}{l}\text { Quercetin-3-O-beta-xyloside } \\
\text { (Reynoutrin; Quercetin } \\
\text { 3-O-Beta-D-Xylopyranoside) }\end{array}$ & $\mathrm{C}_{20} \mathrm{H}_{17} \mathrm{O}_{11}$ & 433.3424 & & 434.90 & & 302.94 & $256.92 ; 164.96$ & $228.91 ; 159.11$ & Rh. sichotense \\
\hline 48 & $\begin{array}{l}\text { Avicularin (Quercetin } \\
\text { 3-Alpha-L- } \\
\text { Arabinofuranoside; } \\
\text { Avicularoside) }\end{array}$ & $\mathrm{C}_{20} \mathrm{H}_{18} \mathrm{O}_{11}$ & 434.3503 & 433.09 & & & $\begin{array}{c}415.07 ; 335.01 ; \\
176.98\end{array}$ & 397.06; 190.99 & $353.07 ; 253.99$ & $\begin{array}{l}\text { Rh. adamsii; } \\
\text { Rh. sichotense }\end{array}$ \\
\hline 49 & $\begin{array}{c}\text { Pentoside } \\
\text { dihydroquercetin }\end{array}$ & & 436 & 435.16 & & & $\begin{array}{c}416.54 ; 300.99 \\
231.01\end{array}$ & $397.02 ; 205.96$ & $\begin{array}{c}361.11 ; 283.02 \\
188.80\end{array}$ & $\begin{array}{l}\text { Rh. adamsii; } \\
\text { Rh. sichotense }\end{array}$ \\
\hline 50 & $\begin{array}{c}\text { Erithrodiol } \\
\text { [3beta-Erytrodiol] }\end{array}$ & $\mathrm{C}_{30} \mathrm{H}_{50} \mathrm{O}_{2}$ & 442.7168 & 441.12 & & & $\begin{array}{l}425.06 ; 381.05 ; \\
300.03 ; 217.04\end{array}$ & $363.06 ; 246.02$ & $319.08 ; 201.02$ & Rh. adamsii \\
\hline 51 & Uvaol & $\mathrm{C}_{30} \mathrm{H}_{50} \mathrm{O}_{2}$ & 442.7168 & & 443.22 & & $425.01 ; 233.07$ & $407.02 ; 325.01$ & $388.99 ; 231.11$ & $\begin{array}{l}\text { Rh. adamsii; } \\
\text { Rh. sichotense }\end{array}$ \\
\hline 52 & $\begin{array}{l}\text { Quercitrin [Quercetin } \\
\text { 3-L-Rhamnoside; } \\
\text { Quercetrin] }\end{array}$ & $\mathrm{C}_{21} \mathrm{H}_{20} \mathrm{O}_{11}$ & 448.3769 & & 448.89 & & $370.95 ; 282.93$ & $352.95 ; 176.98$ & $\begin{array}{c}334.90 ; 222.92 \\
176.97\end{array}$ & Rh. adamsii \\
\hline 53 & Catechin-7-O-glucoside & $\mathrm{C}_{21} \mathrm{H}_{24} \mathrm{O}_{11}$ & 452.4087 & & 453.17 & & $\begin{array}{l}435.15 ; 336.07 \\
209.06\end{array}$ & $\begin{array}{l}\text { 417.16; } 336.11 \\
\quad 226.12\end{array}$ & 209.09 & Rh. sichotense \\
\hline 54 & Micromeric acid & $\mathrm{C}_{30} \mathrm{H}_{46} \mathrm{O}_{3}$ & 454.6844 & & 455.05 & & $408.98 ; 246.98$ & $391.05 ; 287.96$ & 250.96 & Rh. adamsii \\
\hline 55 & $\begin{array}{l}\text { Hyperoside (Quercetin } \\
\text { 3-O- galactoside; } \\
\text { Hyperin) }\end{array}$ & $\mathrm{C}_{21} \mathrm{H}_{20} \mathrm{O}_{12}$ & 464.3763 & & 465.02 & & 302.91 & $256.94 ; 190.87$ & $228.96 ; 172.75$ & Rh. sichotense \\
\hline 56 & $\begin{array}{l}\text { Quercetin 3-O-glucoside } \\
\text { [Isoquercitrin] }\end{array}$ & $\mathrm{C}_{21} \mathrm{H}_{20} \mathrm{O}_{12}$ & 464.3763 & & 465.08 & & 447.00 & 386.96 & $369.12 ; 172$ & Rh. sichotense \\
\hline 57 & $\begin{array}{c}\text { Alpha.-Tocopherol-Beta-D- } \\
\text { Mannoside [Dihydro-2H- } \\
\text { Chromen-6-YI } \\
\text { Hexofuranoside] }\end{array}$ & $\mathrm{C}_{35} \mathrm{H}_{60} \mathrm{O}_{7}$ & 592.8467 & & 593.11 & & 533.08 & 461.10 & 433.11 & Rh. sichotense \\
\hline
\end{tabular}


Figures 2-11 shows examples of the decoding spectra (collision-induced dissociation (CID) spectrum) of the ion chromatogram obtained using tandem mass spectrometry. The CID spectrum in negative ion modes of fraxetin-7-O-beta-glucoronide from $R h$. adamsii and $R h$. sichotense are shown in Figures 2 and 3.

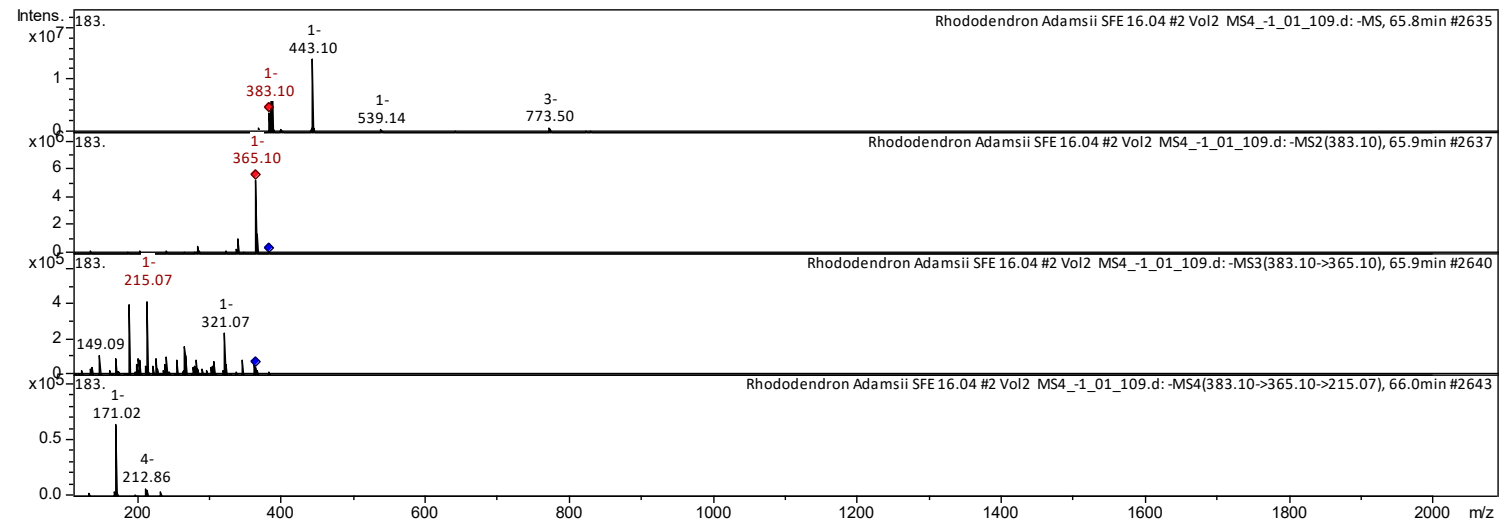

Figure 2. Collision-induced dissociation (CID) spectrum of fraxetin-7-O-beta-glucoronide from Rh. adamsii, $m / z$ 383.10.

The $[\mathrm{M}-\mathrm{H}]^{-}$ion produced fragment ion with $\mathrm{m} / \mathrm{z} 383.10$ (Figure 2). The fragment ion with $\mathrm{m} / \mathrm{z}$ 383.10 produced characteristic daughter ion with $\mathrm{m} / \mathrm{z} 321.07, \mathrm{~m} / \mathrm{z} 215.07$ and $\mathrm{m} / \mathrm{z} 149.09$. The fragment ion with $\mathrm{m} / \mathrm{z} 215.07$ formed two daughter ions with $\mathrm{m} / \mathrm{z} 171.02, \mathrm{~m} / \mathrm{z} 212.86$. It was identified in the bibliography in extract from rhododendron L. palustre [13,30-33,45].

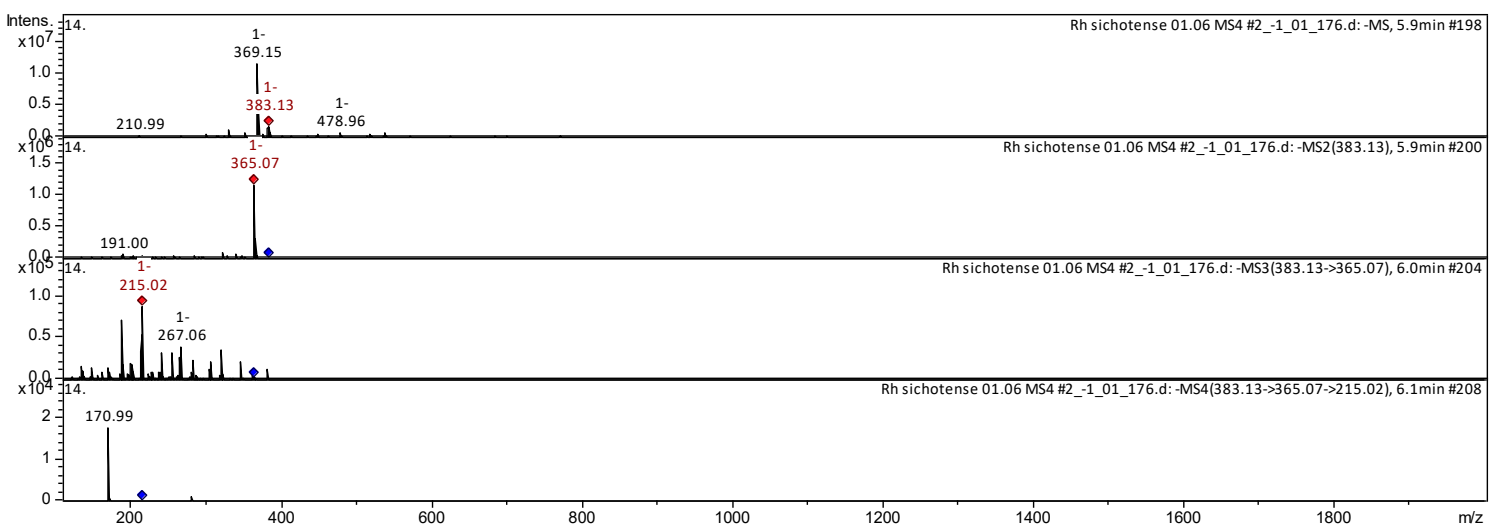

Figure 3. CID spectrum of fraxetin-7-O-beta-glucoronide from $R h$. sichotense, $m / z$ 383.13.

The $[\mathrm{M}-\mathrm{H}]^{-}$ion produced fragment ion with $\mathrm{m} / \mathrm{z} 383.13$ (Figure 3). The fragment ion with $\mathrm{m} / \mathrm{z}$ 383.13 produced two fragments with $\mathrm{m} / \mathrm{z} 365.07, \mathrm{~m} / \mathrm{z}$ 191.00. The fragment ion with $\mathrm{m} / \mathrm{z} 365.07$ produced two characteristic daughter ions with $m / z 267.06$ and $m / z 215.02$. The fragment ion with $\mathrm{m} / z 215.02$ formed a daughter ion with $\mathrm{m} / \mathrm{z}$ 170.99. It was identified in the bibliography of the methanolic extract from rhododendron L. palustre [30-33,44].

The CID spectrum in positive ion modes of Beta-sitosterin from $R h$. adamsii and $R h$. sichotense is shown in Figures 4 and 5. 


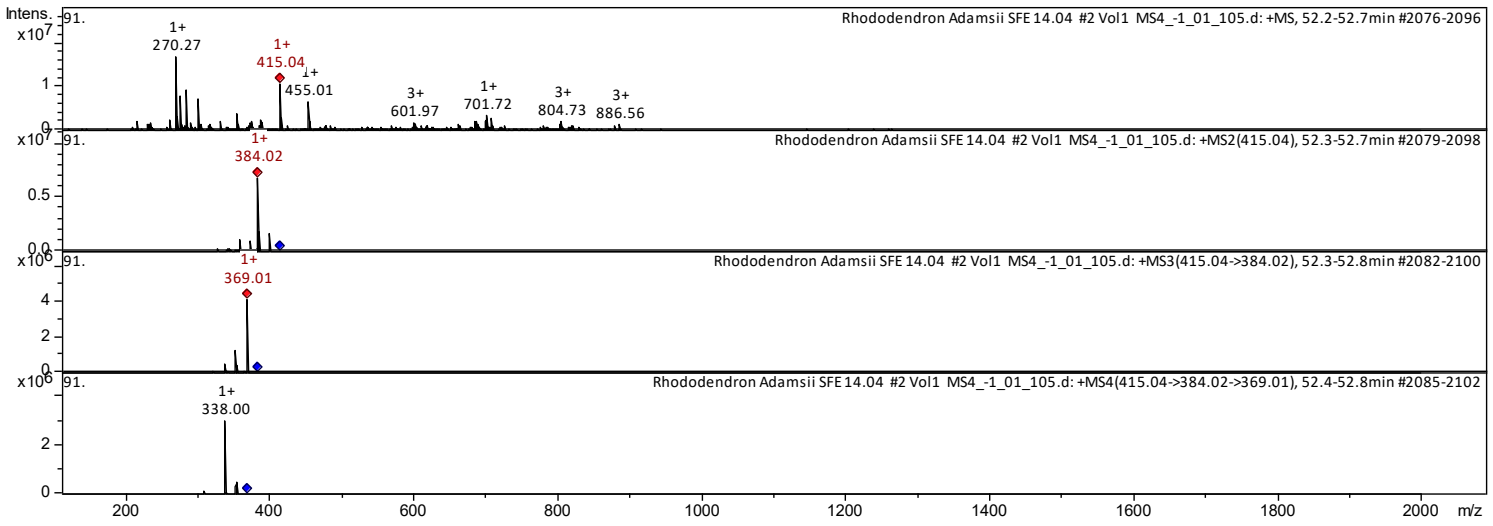

Figure 4. CID spectrum of Beta-sitosterin from Rh. adamsii, $m / z 415.04$

The $[\mathrm{M}+\mathrm{H}]^{+}$ion produced one fragment with $m / z 415.04$ (Figure 4). The fragment ion with $\mathrm{m} / \mathrm{z} 384.02$ produced one daughter ion with $\mathrm{m} / \mathrm{z} 369.01$. The fragment ion with $\mathrm{m} / \mathrm{z} 369.01$ formed a daughter ion with $\mathrm{m} / \mathrm{z}$ 338.00. It was identified in the bibliography in the extract from rhododendrons L. palustre [30-33,41,44,45] and Rh. adamsii $[10,12,15,16,42,52]$.

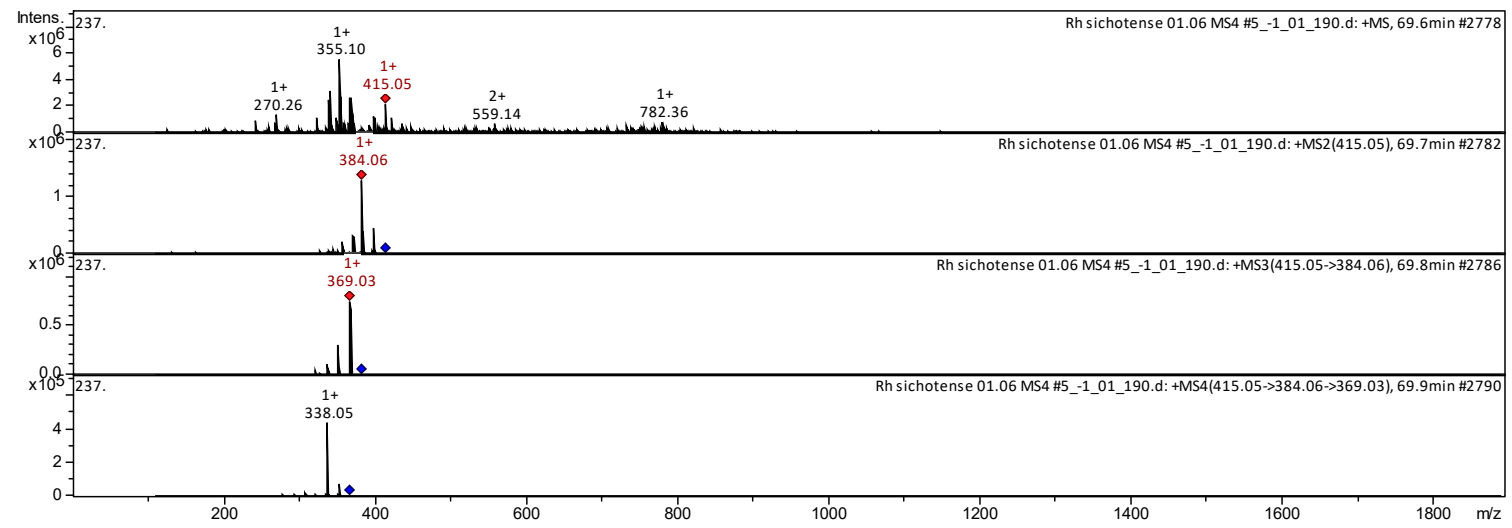

Figure 5. CID spectrum of Beta-sitosterin from $R h$. sichotense, $m / z$ 415.05.

The $[\mathrm{M}+\mathrm{H}]^{+}$ion produced one fragment with $m / z 384.06$ (Figure 5). The fragment ion with $\mathrm{m} / \mathrm{z} 384.06$ produced one daughter ion with $\mathrm{m} / \mathrm{z} 369.03$. The fragment ion with $\mathrm{m} / \mathrm{z} 369.03$ formed a daughter ion with $m / z$ 338.05. It was identified in the bibliography in the extract from rhododendrons L. palustre [30-33,41,44,45] and $R h$. adamsii $[10,12,15,16,42,52]$.

The CID spectrum in negative ion modes of quercetin from $R h$. adamsii and $R h$. sichotense is shown in Figures 6 and 7.

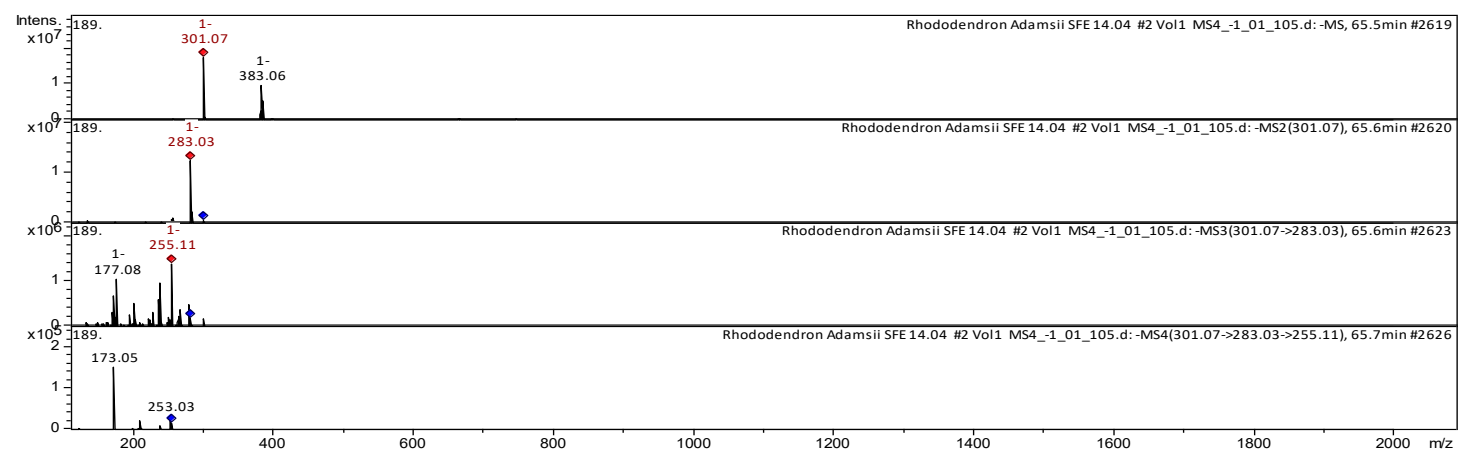

Figure 6. CID spectrum of quercetin from Rh. adamsii, $m / z$ 301.07. 
The $[\mathrm{M}-\mathrm{H}]^{-}$ion produced one fragment ion with $m / z 283.03$ (Figure 6). The fragment ion with $m / z 283.03$ produced two daughter ions with $\mathrm{m} / \mathrm{z} 255.11$ and $\mathrm{m} / \mathrm{z}$ 177.08. The fragment ion with $\mathrm{m} / \mathrm{z}$ 255.11 formed two daughter ions with $\mathrm{m} / \mathrm{z} 253.03$ and $\mathrm{m} / \mathrm{z}$ 173.05. It was identified in the bibliography in extracts from rhododendrons $R h$. sichotense, Rh. micronulatum [38-40]; Rh. ungernii [34]; Rhodiola crenulata [36]; Rh. adamsii [10,12,15,16,42,52]; Rh. parvifolium [10]; Ocimum [43].

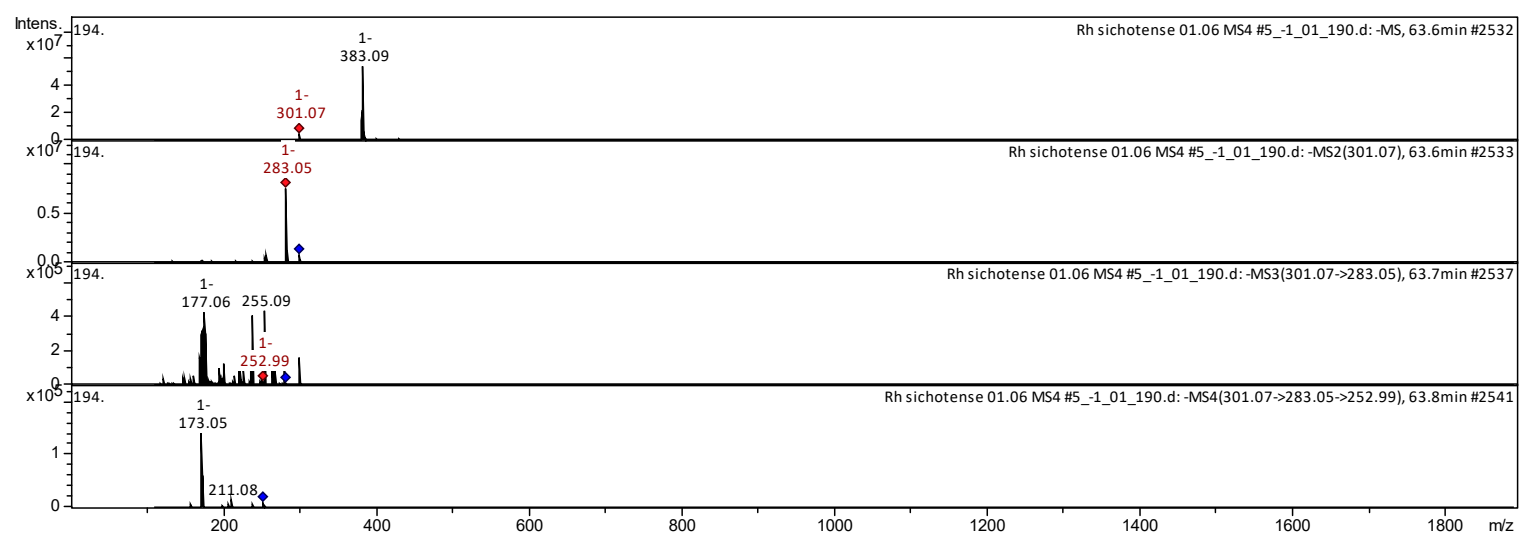

Figure 7. CID spectrum of quercetin from Rh. sichotense, $m / z$ 301.07.

The $[\mathrm{M}-\mathrm{H}]^{-}$ion produced one fragment ion with $m / z 283.03$ (Figure 7). The fragment ion with $m / z 283.03$ produced three daughter ions with $m / z 255.09, m / z 177.06$ and $m / z 252.99$. The fragment ion with $m / z 252.99$ formed two daughter ions with $m / z 211.08$ and $m / z$ 173.05. It was identified in the bibliography in extracts from rhododendrons $R h$. sichotense, Rh. micronulatum [38-40]; Rh. ungernii [34]; Rhodiola crenulata [36]; Rh. adamsii [10,12,15,16,42,52]; Rh. parvifolium [10]; Ocimum [43].

The CID spectrum in negative ion modes of myricetin from $R h$. adamsii and $R h$. sichotense is shown in Figures 8 and 9.

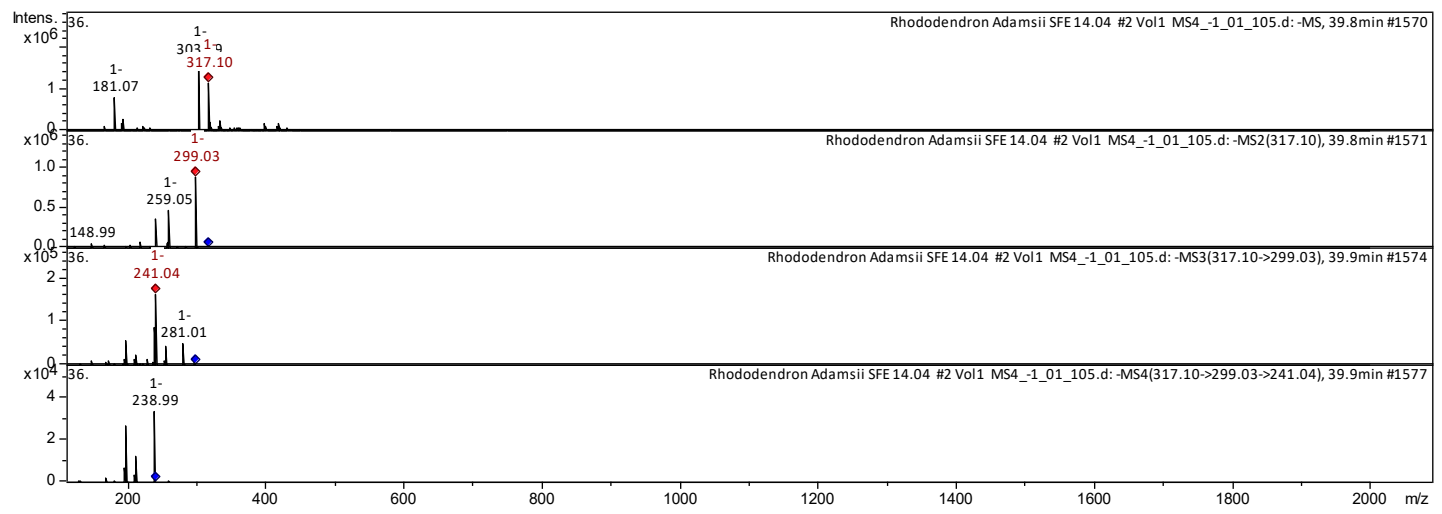

Figure 8. CID spectrum of myricetin from Rh. adamsii, $m / z$ 317.10.

The $[\mathrm{M}-\mathrm{H}]^{-}$ion produced three fragment ions with $\mathrm{m} / \mathrm{z} 299.03, \mathrm{~m} / \mathrm{z} 259.05$ and $\mathrm{m} / \mathrm{z} 148.99$ (Figure 8). The fragment ion with $\mathrm{m} / \mathrm{z} 299.03$ produced two daughter ions with $\mathrm{m} / \mathrm{z} 241.04$ and $\mathrm{m} / \mathrm{z}$ 281.01. The fragment ion with $m / z 241.04$ formed a daughter ion with $m / z 238.99$. It was identified in the bibliography in extracts from rhododendrons $R h$. sichotense, Rh. micronulatum [39-41]; Rh. ungernii [35]; Rhodiola crenulata [37]; Rh. adamsii [16,17,43,53]; Rh. parvifolium [41]; Ocimum [44]. 


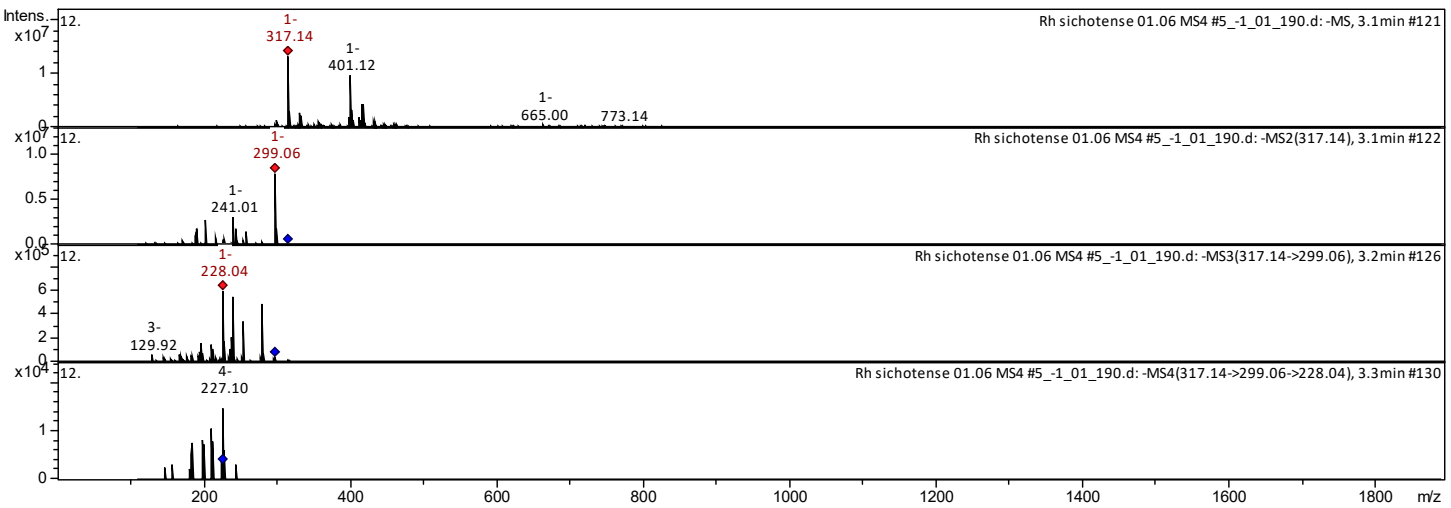

Figure 9. CID spectrum of myricetin from $R h$. sichotense, $m / z$ 317.14.

The $[\mathrm{M}-\mathrm{H}]^{-}$ion produced two fragment ions with $m / z 299.06, m / z 241.01$ (Figure 9). The fragment ion with $\mathrm{m} / \mathrm{z} 299.06$ produced two daughter ions with $\mathrm{m} / \mathrm{z} 228.04$ and $\mathrm{m} / \mathrm{z}$ 129.92. The fragment ion with $\mathrm{m} / \mathrm{z} 228.04$ formed a daughter ion with $\mathrm{m} / \mathrm{z} 227.10$. It was identified in the bibliography in extracts from rhododendrons Rh. sichotense [4,5]; Rh. ungernii [34]; Rhodiola crenulata [36]; Rh. adamsii [10,15,16,42,52]; Rh. parvifolium $[10,40]$.

The CID spectrum in positive ion modes of farrerol from $R h$. adamsii and $R h$. sichotense is shown in Figures 10 and 11. The $[\mathrm{M}+\mathrm{H}]^{+}$ion produced three fragment ions with $m / z 283.02, m / z 244.99$ and $m / z 162.98$ (Figure 10). The fragment ion with $m / z 283.02$ produced two daughter ions with $m / z$ 240.98 and $m / z$ 163.01. The fragment ion with $m / z 240.98$ formed a daughter ion with $m / z$ 170.96. It was identified in the bibliography in extracts from rhododendrons $R h$. dauricum [38-41]; Rh. ungernii [34].

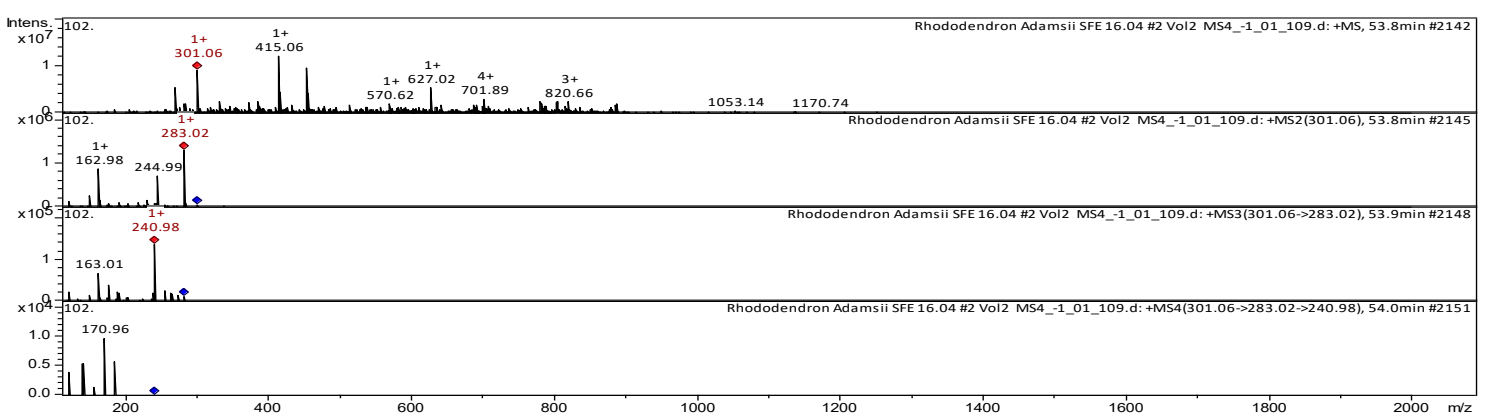

Figure 10. CID spectrum of farrerol from $R h$. adamsii, $m / z$ 301.06.

The $[\mathrm{M}+\mathrm{H}]^{+}$ion produced two fragment ions with $\mathrm{m} / \mathrm{z} 282.95$ and $\mathrm{m} / \mathrm{z} 180.98$ (Figure 11).

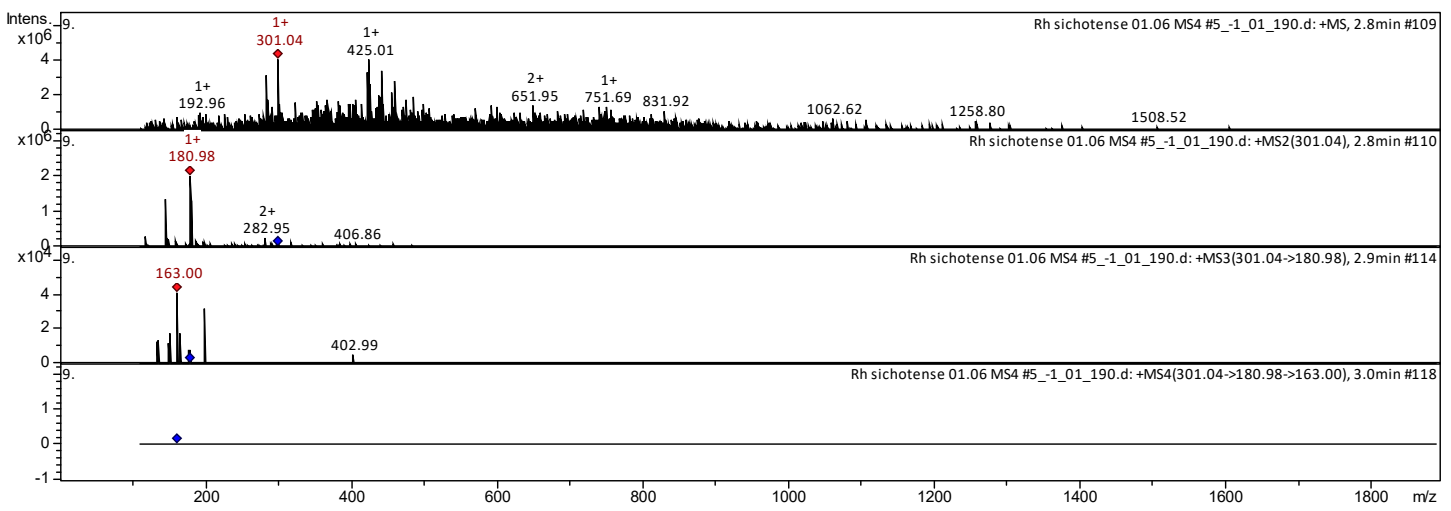

Figure 11. CID spectrum of farrerol from $R h$. sichotense, $m / z 301.06$. 
The fragment ion with $\mathrm{m} / \mathrm{z} 180.98$ formed a daughter ion with $\mathrm{m} / \mathrm{z}$ 163.00. It was identified in the bibliography in extracts from rhododendrons Rh. dauricum [38-40]; Rh. ungernii [34].

\section{Materials and Methods}

\subsection{Materials}

The objects of study were purchased samples of $R h$. sichotense (leaves and stems) from Primorsky Krai (the eastern slope of the Sikhote Alin ridge) and Rh. adamsii (leaves and stems) from the area near lake Baykal, Russia. All samples were morphologically authenticated according to the current standard of Russian Pharmacopeia [54]. All samples were immediately washed weighed by $10 \mathrm{~g}$ aliquot then frozen and kept until extraction.

\subsection{Chemicals and Reagents}

HPLC-grade acetonitrile was purchased from Fisher Scientific (Southborough, UK), MS-grade formic acid was from Sigma-Aldrich (Steinheim, Germany). Ultra-pure water was prepared from a SIEMENS ULTRA clear (SIEMENS water technologies, Munich, Germany), and all other chemicals were analytical grade.

\subsection{Liquid Chromatography}

HPLC was performed using a Shimadzu LC-20 Prominence HPLC (Kanda-Nishikicho 1-chrome, Shimadzu, Chiyoda-ku, Tokyo, Japan), equipped with a UV-sensor and a Shodex ODP-40 4E $(250 \times 4.6 \mathrm{~mm}$, particle size: $4 \mu \mathrm{m})$ reverse phase $\mathrm{C} 18$ column to perform the separation of multicomponent mixtures. The gradient elution program was as follows: $0.01-4 \mathrm{~min}, 100 \% \mathrm{~A} ; 4-60 \mathrm{~min}, 100-25 \% \mathrm{~A}$; 60-75 $\mathrm{min}, 25-0 \% \mathrm{~A}$; control washing 75-120 $\mathrm{min} 0 \%$ A. The entire HPLC analysis was performed using a UV-VIS detector SPD-20A (Kanda-Nishikicho 1-chrome, Shimadzu, Chiyoda-ku, Tokyo, Japan) at wavelengths of 230 and $330 \mathrm{~nm}$, at $17^{\circ} \mathrm{C}$ provided with a column oven CTO-20A (Kanda-Nishikicho 1-chrome, Shimadzu, Chiyoda-ku, Tokyo, Japan) with an injection volume of $20 \mu \mathrm{L}$.

\section{4. $\mathrm{SC}-\mathrm{CO}_{2}$ Extraction}

SC- $\mathrm{CO}_{2}$ extraction was performed using the Supercritical fluid system -500 (Thar SCF Waters, Milford, MA, USA) supercritical pressure extraction apparatus. System options include: Co-solvent pump (Thar Waters P-50 High Pressure Pump), for extracting polar samples. $\mathrm{CO}_{2}$ flow meter (Siemens, Munich, Germany), to measure the amount of $\mathrm{CO}_{2}$ being supplied to the system, multiple extraction vessels, to extract different sample sizes or to increase the throughput of the system. Flow rate was 50 $\mathrm{mL} / \mathrm{min}$ for liquid $\mathrm{CO}_{2}$ and $1.00 \mathrm{~mL} / \mathrm{min}$ for EtOH. Samples for extraction of $10 \mathrm{~g}$ of frozen Rh. sichotense and $R h$. adamsii pre-cut into pieces no more than $1 \mathrm{~cm}$ were used. Several experimental conditions were investigated, working in a pressure range of $300-400$ bar, with $1 \%$ of $\mathrm{C}_{2} \mathrm{H}_{5} \mathrm{OH}$ as co-solvent and a temperature range of $50-60{ }^{\circ} \mathrm{C}$. The extraction time was counted after reaching the working pressure and equilibrium flow, and it was 60-70 min for each sample.

\subsection{Mass Spectrometry}

MS analysis was performed on an ion trap amaZon SL (BRUKER DALTONIKS, Bremen, Germany) equipped with an ESI source in negative ion mode. The optimized parameters were obtained as follows: ionization source temperature: $70{ }^{\circ} \mathrm{C}$, gas flow: $4 \mathrm{~L} / \mathrm{min}$, nebulizer gas (atomizer): $7.3 \mathrm{psi}$, capillary voltage: $4500 \mathrm{~V}$, end plate bend voltage: $1500 \mathrm{~V}$, fragmentary: $280 \mathrm{~V}$, collision energy: $60 \mathrm{eV}$ MS/MS MS 4 (four stages of separation). An ion trap was used in the scan range $m / z$ 100-1700 for MS and the capture rate was one spectrum/s for MS and two spectrum/s for MS/MS. All experiments were repeated three times. A four-stage ion separation mode (MS/MS mode) was implemented. 


\section{Conclusions}

Aiming to optimize the extraction of target analytes from the $R h$. sichotense and $R h$. adamsii leaves and stems, several experimental conditions were investigated working in a pressure range of 300-400 bar, with $1 \%$ of $\mathrm{C}_{2} \mathrm{H}_{5} \mathrm{OH}$ as co-solvent and a temperature range of $50-60{ }^{\circ} \mathrm{C}$. Although this approach is not quantitative for evaluation of each analyte, it is semiquantitative when comparing a series of extractions and allows better comparison of the yield without loss of individual analytes during fractionation and sample preparation. The best results were obtained at 370 bar and $60{ }^{\circ} \mathrm{C}$.

High-accuracy mass spectrometric data were recorded on an ion trap amaZon SL BRUKER DALTONIKS equipped with an ESI source in the negative ion mode. The four-stage ion separation mode was implemented to perform correct identification. Under these conditions a total of 800 peaks were detected in the ion chromatogram. An optimized extraction process with $\mathrm{SC}-\mathrm{CO}_{2}$ (co-solvent $1 \%$ ethanol) provided the samples for an accurate analytical study by HPLC-MS/MS technique. A total of 50 different biologically active components were identified in the $R h$. adamsii SC-CO $\mathrm{CO}_{2}$ extracts. A total of 30 different biologically active components were identified in the $R h$. sichotense $\mathrm{SC}-\mathrm{CO}_{2}$ extracts.

An analysis of the similarity of the composition of biologically active components of $R h$. sichotense and $R h$. adamsii revealed their significant relationship. An analysis of morphometric parameters and composition of $R h$. sichotense and $R h$. adamsii flavonoids from populations of the Far East and Siberia indicated the presence of ecological and geographical variability. Inter-population differences in morphometric indicators are more significant than in chemical ones. Moreover, the former is more associated with climatic factors, while the latter are more associated with edaphic growth factors. The Rh. adamsii extract was more diverse in chemical composition than Rh. sichotense. A particularly large difference was observed in acidic components such as caffeic acid, azelaic acid, myristic acid, pentadecanoic acid, palmitoleic acid, linoleic acid. The extract of $R h$. sichotense contained mainly stearic acid, nonadecanoic acid, montanic acid, behenic acid, tetracosanoic acid and chlorogenic acid. The flavonoid content of both rhododendrons was mainly the same.

These data could support future research for the production of a variety of pharmaceutical products containing ultra-pure SC- $\mathrm{CO}_{2}$ extracts of $R h$. sichotense and $R h$. adamsii. The richness of various biologically active compounds, including flavonoids: quercetin, kaempferol, dihydroquercetin, farrerol, myricetin, etc. provides great opportunities for the design of new drugs based on extracts from this species of rhododendron.

Supplementary Materials: The following are available online, Figure S1: Chemical profiles of the Rh. adamsii sample represented ion chromatogram from SC- $\mathrm{CO}_{2}$ extract; Figure S2: Chemical profiles of the Rh. sichotense sample represented ion chromatogram from $\mathrm{SC}-\mathrm{CO}_{2}$ extract.

Author Contributions: Conceptualization, A.Z. and K.G.; methodology, A.Z., M.R.; software, M.R.; validation, M.R., K.G.; formal analysis, M.R., A.Z., V.G.; investigation, M.R. and A.Z.; resources, K.G. and A.Z.; data curation, K.G.; writing—original draft preparation-M.R., A.Z., S.E.; writing—review and editing A.Z., V.G., S.E. and K.G.; visualization, M.R., A.Z., S.E. and V.G.; supervision, K.G.; project administration, A.Z., K.G. All authors have read and agreed to the published version of the manuscript.

Funding: This research was funded by Council on Grants of the President of the Russian Federation (СП3156.2019.4).

Conflicts of Interest: The authors declare no conflict of interest.

\section{References}

1. Pojarkova, A.I. Genus Ericaceae, D.K.-vacciniaceous. Flora USSR 1952, 18, 26-93. Available online: https://docviewer.yandex.ru/view/0/?page=1\&*=G3rUdUDjrwwhelX3asXdHS\%2Fw9897InVybCI6InlhL WRpc2stcHVibGljOi8vN0x0R01ZV1VYaEhFcmRwdTFtTmprVnJoNGl4dmhDMDhsbVBpcjROcjVnaz0iLC J0aXRsZSI6ItCk0JvQntCg0JBf0KHQodCh0KBf0KIxOF8xOTUyLmRqdnUiLCJub2lmcmFtZSI6ZmFsc2UsI nVpZCI6IjAiLCJ0cyI6MTU5NzU4Mjc3MzcwNywieXUiOiI4OTgyMDE4MDYxNTU5MDQ4Mzk5In0\%3D (accessed on 29 June 2020). (In Russian) 
2. Aleksandrova, M.S. Rhododendrons of Natural Flora of the USSR; Nauka: Moscow, Russia, 1975; p. 112. Available online: https://docplayer.ru/42008573-Rododendrony-prirodnoy-flory-sssr.html (accessed on 29 June 2020). (In Russian)

3. Zaytseva, G.Y.; Ambros, E.V.; Karakulov, A.V.; Novikova, T.I. Flow cytometric determination of genome size and ploidy level of some frost-resistant cultivars and species of Rhododendron L. native to Asian Russia. Botanica Pacifica. Bot Pac. 2018, 7, 97-100. [CrossRef]

4. Belousova, N.I.; Khan, V.A.; Tkachev, A.V. The chemical composition of essential oil of Rhododendron. Khimiya Rastitel'nogo Syr'iya (Chem. Plant Raw Mater.) 1999, 3, 5-38. Available online: https://elibrary.ru/item .asp?id=9444189 (accessed on 29 June 2020). (In Russian).

5. Belousov, M.V.; Komissarenko, N.F.; Berezovskaya, T.P.; Tochkova, T.V. Content of flavonoids and coumarins in the Siberian-Far Eastern species of the Ericaceae family. Rastit. Resur. 1994, 4, 44-47. Available online: https://www.elibrary.ru/item.asp?id=20162300 (accessed on 29 June 2020). (In Russian).

6. Varlygina, T.I.; Kamelin, R.V.; Kiseleva, K.V. Red Data Book of Russian Federation; KMK: Moskow, Russia, 2008; p. 855. ISBN 978-5-87317-476-8.

7. Firsov, G.A.; Egorov, A.A.; Byalt, V.V.; Neverovsky, V.J.; Orlova, L.V.; Volchanskaya, A.V.; Lavrentyev, N.V. Arboreal plants of the Red Data Book of Russia in collection of Saint Petersburg Forest-Technical Academy. Hortus Botanicus. 2010, 5, 1-16. Available online: https://www.elibrary.ru/item.asp?id=21827419 (accessed on 29 June 2020). [CrossRef]

8. Khokhryakov, A.P.; Mazurenko, M.T. Vascular plants of the Soviet Far East. Science 1991, 5, 119. Available online: https://www.elibrary.ru/author_items.asp (accessed on 29 June 2020). (In Russian).

9. Hubich, A.I.; Puchkova, K.V.; Zalesskaya, N.A.; Kryuchkova, N.V. The investigation of the adaptogenic properties of Rhododendron adamsii Rehder. on experimental models in vivo. J. Belarus. State Univ. Biol. 2018, 1, 60-68. (In Russian)

10. Mirovich, V.M.; Konenkina, T.A.; Fedoseeva, G.M. Qualitative structure od essential oil of Rhododendron adamsii and parvifolium, growing in East Siberia. Siberian Med. J. 2008, 76, 79-82. Available online: https://www.elibrary.ru/item.asp?id=17844859 (accessed on 29 June 2020). (In Russian).

11. Belousova, N.I.; Khan, V.A. Bicyclic monoterpenoids of the essential oil of Ledum palustre. Chem. Nat. Compd. 1990, 5, 627-629.

12. Kurshakova, G.V.; Fedorov, A.A.; Yakimov, P.A. Some data on the chemical composition and pharmacological effect of rhododendron Adams-Rhododendron adamsii Rend. Trudy Botanicheskogo instituta im. V. L. Komarova AN SSSR 1961, V, 216-220. (In Russian)

13. Belousov, M.V.; Berezovskaya, T.P.; Komissarenko, N.F.; Tikhonova, L.A. Flavonoids of Siberian and Far-Eastern species of rhododendrons of the subsgenus Rhodorastrum. Chem. Nat. Compd. 1998, 34, 510-511. [CrossRef]

14. Fini, A.; Brunetti, C.; Di Ferdinando, M.; Ferrini, F.; Tattini, M. Stress-induced flavonoid biosynthesis and the antioxidant machinery of plants. Plant. Signal. Behav. 2011, 6, 709-711. [CrossRef] [PubMed]

15. Rogachev, A.D. Phytochemical study of Rhododendron Adamsii Rheder. Ph.D.'s Thesis, Novosibirsk University, Novosibirsk, Russia, 2009. (In Russian)

16. Rogachev, A.D.; Fomenko, V.V.; Sal'nikova, O.I.; Pokrovskii, L.M.; Salakhutdinov, N.F. Comparative analysis of essential oil compositions from leaves and stems of Rhododendron adamsii, R. aureum, and R. dauricum. Chem. Nat. Compd. 2006, 42, 426-430. [CrossRef]

17. Razgonova, M.P.; Zacharenko, A.M.; Kalenik, T.K.; Nosyrev, A.E.; Stratidakis, A.K.; Mezhuev, Y.O.; Burykina, T.I.; Nicolae, A.C.; Arsene, A.L.; Tsatsakis, A.M.; et al. Supercritical fluid technology and supercritical fluid chromatography for application in ginseng extracts. Farmacia 2019, 67, 202-212. [CrossRef]

18. Razgonova, M.; Zakharenko, A.; Shin, T.-S.; Chung, G.; Golokhvast, K. Supercritical $\mathrm{CO}_{2}$ Extraction and Identification of Ginsenosides in Russian and North Korean Ginseng by HPLC with Tandem Mass Spectrometry. Molecules 2020, 25, 1407. [CrossRef] [PubMed]

19. Morozov, Y.A.; Pupykina, K.A.; Blagorazumnaya, N.V.; Aliev, A.M.; Morozova, E.V. Comparative analysis of carbon dioxide extracts from plant material of Schisandra chinensis: Leaves, woody stems, rhizomes with roots. Med. Bull. Bashkortostan. 2018, 13, 46-51.

20. Aliev, A.M.; Radjabov, G.K.; Musaev, A.M. Dynamics of supercritical extraction of biological active substances from the Juniperus communis var. saxatillis. J. Supercrit. Fluids 2015, 102, 66-72. [CrossRef] 
21. Rovetto, L.J.; Aieta, N.V. Supercritical carbon dioxide extraction of cannabinoids from Cannabis sativa L. J. Supercrit. Fluids 2017, 129, 16-27. [CrossRef]

22. Baldino, L.; Della Porta, G.; Sesti Osseo, L.; Reverchon, E.; Adami, R. Concentrated oleuropein powder from olive leaves using alcoholic extraction and supercritical $\mathrm{CO}_{2}$ assisted extraction. J. Supercrit. Fluids. 2018, 133, 65-69. [CrossRef]

23. Mehariya, S.; Iovine, A.; Di Sanzo, G.; Larocca, V.; Martino, M.; Leone, G.P.; Casella, P.; Karatza, D.; Marino, T.; Musmarra, D.; et al. Supercritical fluid extraction of lutein from Scenedesmus almeriensis. Molecules 2019, 24, 1324. [CrossRef]

24. Leone, G.P.; Balducchi, R.; Mehariya, S.; Martino, M.; Larocca, V.; Di Sanzo, G.; Iovine, A.; Casella, P.; Marino, T.; Karatza, D.; et al. Selective Extraction of $\omega-3$ Fatty Acids from Nannochloropsis sp. Using Supercritical $\mathrm{CO}_{2}$ extraction. Molecules 2019, 24, 2406. [CrossRef] [PubMed]

25. Senica, M.; Stampar, F.; Miculic-Petkovsek, M. Different extraction processes affect the metabolites in blue honeysuckle (Lonicera caerulea L. subsp. edulis) food products. Turk. J. Agric. For. 2019, 43, 576-585. [CrossRef]

26. Colak, A.M.; Okatan, V.; Polat, M.; Guclu, S.F. Different harvest times affect market quality of Lycium barbarum L. berries. Turk. J. Agric. For. 2019, 43, 326-333. [CrossRef]

27. Baldino, L.; Reverchon, E. Challenges in the production of pharmaceutical and food related compounds by $\mathrm{SC}-\mathrm{CO}_{2}$ processing of vegetable matter. J. Supercrit. Fluids 2018, 134, 269-273. [CrossRef]

28. Popova, A.S.; Ivahnov, A.D.; Skrebets, T.E.; Bogolitsyn, K.G. Supercritical fluid extraction of carotenoids and chlorophyll from Ledum palustre. Khimiya Rastitel'nogo Syr'iya (Chem. Veg. Raw Mater.) 2018, 1, 61-66. (In Russian)

29. Baananou, S.; Bagdonaite, E.; Marongiu, B.; Piras, A.; Porcedda, S.; Falconieri, D.; Boughattas, N.A. Supercritical $\mathrm{CO}_{2}$ extract and essential oil of aerial part of Ledum palustre L.-Chemical composition and anti-inflammatory activity. Nat. Prod. Res. 2015, 29, 999-1005. [CrossRef]

30. Bukreyeva, T.V.; Shavarda, A.L.; Matusevich, O.V.; Morozov, M.A. Ursane, oleanane, lupine triterpenoids from leaves of Ledum palustre (Ericaceae) from North-West Russia. Rastit. Resur. 2013, 49, 395-403. (In Russian)

31. Butkiene, R.; Sakociute, V.; Latvenaite, D.; Mockute, D. Composition of young and aged shoot essential oils of the wild Ledum palustre L. Chemija 2008, 19, 19-24.

32. Buzuk, A.G.; Buzuk, G.N. The study of chemical variability of essential oil composition of Ledum palustre L., growing on the territory of the republic of Belarus. Vestnik Farmacii. 2016, 4, 18-25. (In Russian)

33. Dampc, A.; Luczkiewicz, M. Rhododendron tomentosum (Ledum palustre). A Review of traditional use based on current research. Fitoterapia 2013, 85, 130-143. [CrossRef]

34. Dede, E.; Genc, N.; Elmastas, M.; Aksit, H.; Erenler, R. Chemical constituents Isolated from Rhododendron ungernii with Antioxidant Profile. Nat. Prod. J. 2019, 9, 238-243. [CrossRef]

35. Ganina, M.M. and Popova, O.I. Content of phenolic compounds in shoots of Ledum procumbent (Ledum decumbens Lodd. ex Steud) growing on the territory of the Yamalo-nenets autonomous district. Khim.-Farm. Zh. 2015, 49, 33-35.

36. Han, F.; Li, Y.; Ma, L.; Liu, T.; Wu, Y.; Hu, R.; Song, A.; Yin, R. A rapid and sensitive UHPLC-FT-ICR MS/MS method for identification of chemical constituents in Rhodiola crenulata extract, rat plasma and rat brain after oral administration. Talanta 2016, 160, 183-193. [CrossRef] [PubMed]

37. Harborne, J.B.; Williams, C.A. Leaf survey of flavonoids and simple phenols in the genus Rhododendron. Phytochemistry 1971, 10, 2727-2744. [CrossRef]

38. Izotov, D.V.; Tagiltsev, Y.G.; Kolesnikova, R.D.; Tsyupko, V.A. Biologically active substances of Far-Eastern Labrador tea. Lesnoy J. 2010, 2, 24-30. Available online: https://www.elibrary.ru/item.asp?id=15198739 (accessed on 29 June 2020). (In Russian)

39. Karpova, E.A.; Karakulov, A.V. Flavonoids of some Rhododendron species of flora of Siberia and the Far East. Khimiya Rastitel'nogo Syr'ya (Chem. Plant Raw Mater.) 2013, 2, 119-126. Available online: https://www.elibrary.ru/item.asp?id=20332638 (accessed on 29 June 2020). (In Russian).

40. Karakulov, A.V.; Karpova, E.A.; Vasiliev, V.G. Ecological and geographical variation of morphometric parameters and flavonoid composition of Rhododendron parvifolium. Turczaninowia 2018, 21, 133-144.

41. Korotaeva, M.S.; Belousov, M.V.; Fursa, N.S. Flavonoids and hydroxycinnamic acids content in Ledum palustre (Ericaceae) above-ground part. Rastit. Resur. 2008, 44, 66-75. Available online: https://www.elibrary.ru/item.a sp?id=9940517 (accessed on 29 June 2020). (In Russian) 
42. Mirovich, V.M.; Fedoseeva, G.M.; Zjubr, T.P.; Fedoseev, A.P.; Paisova, O.I.; Kuklina, L.B. Elaboration of the method of receipt of the dry extract from sprouts of Rhododendron adamsii, having actoprotective and antimicrobic activity. Sibirskii medicinskii Zhurnal. 2006, 9, 96-98. (In Russian)

43. Pandey, R.; Kumar, B. HPLC-OTOF-MS/MS-based rapid screening of phenolics and triterpenic acids in leaf extracts of Ocimum species and heir interspecies variation. J. Liq. Chromatogr. Relat. Technol. 2016, 39, 225-238. [CrossRef]

44. Plyashechnik, M.A. Chemical composition of Ledum palustre L. essential oil under increasing nitrogen availability in soils of cryolitzone (Central Evenkia). Khimiya Rastitel'nogo Syr'iya (Chem. Plant Raw Mater.) 2012, 2, 139-144. (In Russian)

45. Raal, A.; Orav, A.; Gretchushnikova, T. Composition of the essential oil of the Rhododendron tomentosum Harmaja from Estonia. Nat. Prod. Res. 2014, 28, 1091-1098. [CrossRef]

46. Suzuki, H.; Sasaki, R.; Ogata, Y.; Nakamura, Y.; Sakurai, N.; Kitajima, M.; Takayama, H.; Kanaya, S.; Aoki, K.; Shibata, D.; et al. Metabolic profiling of flavonoids in Lotus japonicus using liquid chromatography Fourier transform ion cyclotron resonance mass spectrometry. Phytochemistry 2008, 69, 99-111. [CrossRef] [PubMed]

47. Taamalli, A.; Arráez-Román, D.; Abaza, L.; Iswaldi, I.; Fernández-Gutiérrez, A.; Zarrouk, M.; Segura-Carretero, A. LC-MS-based metabolite profiling of methanolic extracts from the medicinal and aromatic species Mentha pulegium and Origanum majorana. Phytochem. Anal. 2015, 26, 320-330. [CrossRef] [PubMed]

48. Ul'yanovskii, N.V.; Kosyakov, D.S.; Pokryshkin, S.A.; Bogolitsyn, K.G.; Ul'yanovskaya, O.S. Study of volatile compounds composition of Ledum palustre L. using the method of thermodesorption gas chromatography -mass spectrometry. Khimiya Rastitel'nogo Syr'iya (Chem. Plant Raw Mater.) 2014, 4, 153-161. (In Russian) [CrossRef]

49. Yang, S.T.; Wu, X.; Rui, W.; Guo, J.; Feng, Y.F. UPLC/Q-TOF-MS analysis for identification of hydrophilic phenolics and lipophilic diterpenoids from Radix Salviae Miltiorrhizae. Acta Pharm. 2015, 27, 711-728. [CrossRef]

50. Zaytseva, N.V.; Pogulyaeva, I.A. Chromatographic Analysis of Chemical Composition of the Genus Rhododendron Plants Growing on the Mountain of Evota (South Yakutia). J. Chem. Chem. Eng. 2014, 8, 516-523.

51. Jin, C.; Strembiski, W.; Kulchytska, Y.; Micetich, R.G.; Daneshtalab, M. Flavonoid glycosides from Ledum palustre L. subsp. decumbens (Ait.) Hulton. DARU. J. Pharm. Sci. 1999, 7, 4.

52. Komarova, N.I.; Rogachev, A.D.; Chernyak, E.I.; Morozov, S.V.; Fomenko, V.V.; Salakhutdinov, N.F. Quantitative HPLC determination of main flavonoid content of Rhododendron adamsii leaves and stems. Chem. Nat. Compd. 2009, 45, 1. [CrossRef]

53. Okhlopkova, Z.M.; Chirikova, N.K. Component composition analysis of essential oil of the Ledum palustre L., growing in Yakutia. Fundam. Res. 2012, 11, 1334-1336.

54. Russian State Pharmacopeia XIII. 2016. Available online: http://pharmacopoeia.ru/en/gosudarstvennaya-far makopeya-xiii-online-gf-13-online/ (accessed on 29 June 2020).

Sample Availability: Not available.

(C) 2020 by the authors. Licensee MDPI, Basel, Switzerland. This article is an open access article distributed under the terms and conditions of the Creative Commons Attribution (CC BY) license (http://creativecommons.org/licenses/by/4.0/). 